\title{
Assessing vertical axis rotations in large-magnitude extensional settings: A transect across the Death Valley extended terrane, California
}

\author{
Michael S. Petronis and John W. Geissman \\ Department of Earth and Planetary Sciences, University of New Mexico, Albuquerque, New Mexico, USA
}

Daniel K. Holm

Department of Geology, Kent State University, Kent, Ohio, USA

Brian Wernicke and Edwin Schauble
Division of Geological and Planetary Sciences, California Institute of Technology, Pasadena, California, USA

Received 11 September 2000; revised 7 May 2001; accepted 14 July 2001; published 18 January 2002.

[1] Models for Neogene crustal deformation in the central Death Valley extended terrane, southeastern California, differ markedly in their estimates of upper crustal extension versus shear translations. Documentation of vertical axis rotations of range-scale crustal blocks (or parts thereof) is critical when attempting to reconstruct this highly extended region. To better define the magnitude, aerial extent, and timing of vertical axis rotation that could mark shear translation of the crust in this area, paleomagnetic data were obtained from Tertiary igneous and remagnetized Paleozoic carbonate rocks along a roughly east-west traverse parallel to about $36^{\circ} \mathrm{N}$ latitude. Sites were established in $\sim 7$ to 5 Ma volcanic sequences (Greenwater Canyon and Brown's Peak) and the $\sim 10 \mathrm{Ma}$ Chocolate Sundae Mountain granite in the Greenwater Range, $\sim 8.5$ to $7.5 \mathrm{Ma}$ and 5 to 4 Ma basalts on the east flank of the Black Mountains, the 10.6 Ma Little Chief stock and upper Miocene(?) basalts in the eastern Panamint Mountains, and Paleozoic Pogonip Group carbonate strata in the north central Panamint Mountains. At the site level, most materials yield readily interpretable paleomagnetic data. Group mean directions, after appropriate structural corrections, suggest no major vertical axis rotation of the Greenwater Range (e.g., $D=359^{\circ}, I=46^{\circ}, \alpha_{95}=8.0^{\circ}$, $N=12(7$ normal $(\mathrm{N}), 5$ reversed (R) polarity sites)), little post-5 Ma rotation of the eastern Black Mountains (e.g., $D=006^{\circ}, I=61^{\circ}, \alpha_{95}=4.0^{\circ}, N=9 \mathrm{~N}, 6 \mathrm{R}$ sites), and no significant post- $10 \mathrm{Ma}$ rotation of the Panamint Range (e.g., $D=181^{\circ}, I=-51^{\circ}, \alpha_{95}=6.5^{\circ}, N=9$ R sites). In situ data from the Greenwater Canyon volcanic rocks, Chocolate Sundae Mountain granite, Funeral Peak basalt rocks, the Little Chief stock, and Paleozoic carbonate rocks (remagnetized) are consistent with moderate south east-side-down tilting of the separate range blocks during northwest directed extension. The paleomagnetic data reported here suggest that the Panamints shared none of the 7 Ma to recent clockwise rotation of the Black Mountains crystalline core, as proposed in recent models for transtensional development of the central Death Valley extended terrane. INDEX TERMS: 1525 Geomagnetism and Paleomagnetism: Paleomagnetism applied to tectonics (regional, global), 1527 Geomagnetism and Paleomagnetism: Paleomagnetism applied to geologic processes, 8109 Tectonophysics: Continental tectonics-extensional (0905), 8015 Structural Geology: Local crustal structure; KEYWORDS: paleomagnetism, Death Valley, continental extension, vertical axis rotation, crustal deformation, plane strain

\section{Introduction}

[2] Geometrically significant vertical axis rotation of crustal fragments during continental extension has been documented in parts of the Basin and Range province through structural studies [e.g., Burchfiel, 1965; Guth, 1981; Snow and Wernicke, 1989]. The first study to document vertical axis rotations in the region based on paleomagnetic data was that of Gillett and Van Alstine [1982] along the Las Vegas Valley shear zone. Over the past decade, paleomagnetic assessment of vertical axis rotations has contributed to our recognition of the three-dimensional kinematics of extension [e.g., Hudson et al., 1998; Holm et al., 1993; Sonder et al.,

Copyright 2002 by the American Geophysical Union. 0148-0227/02/2001JB000239\$09.00
1994; Petronis, 1998; Stamatakos et al., 1998; Wawryzniec et al., 2001]. An increasing body of evidence suggests that statistically significant vertical axis rotations may be a fundamental, albeit potentially local, characteristic of strongly extended continental lithosphere [Snow and Prave, 1994; Hagstrum and Gans, 1989; Hudson and Geissman, 1987; Nelson and Jones, 1987; Janecke et al., 1991].

[3] The Death Valley region, at the southern part of the Walker Lane belt, is widely considered a classic example of diffuse extension and dextral shear (Figure 1) [Burchfiel and Stewart, 1966; Wright and Troxel, 1973; Stewart, 1983; Hamilton, 1988; Wernicke et al., 1988b]. The relative contributions of extension and shear and the role of vertical axis rotation of large range blocks versus local rotations near strike-slip faults, however, are not yet well defined. Clockwise deflections of strati- 


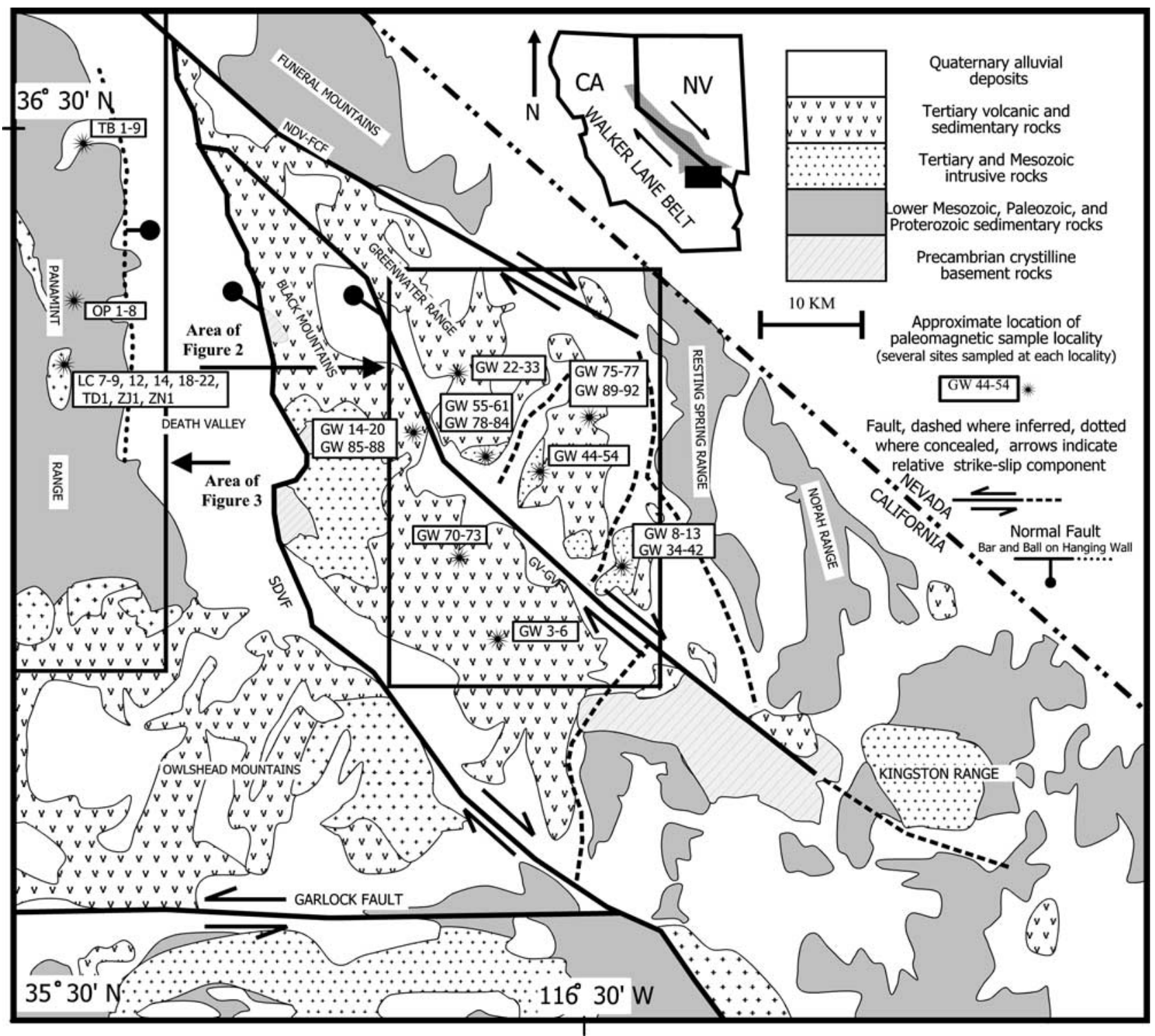

Figure 1. Generalized geologic map of the Death Valley region depicting ranges, major fault zones, and paleomagnetic sample localities used for this study. Stripped regions denote pre-Quaternary rocks; open areas are Quaternary deposits. NDV-FCF, northern Death Valley-Furnace Creek fault; GV-GVF, Greenwater Valley-Grand View fault; SDVF, southern Death Valley fault. Modified from Holm et al. [1994b] and Stewart [1983].

graphic contacts near dextral faults [Burchfiel, 1965; Stewart et al., 1968] and some paleomagnetic evidence for significant clockwise rotations [Rosenbaum et al., 1991; Sonder et al., 1994; Holm et al., 1993] are consistent with distributed dextral shear in the southern Walker Lane, as is the contemporary deformation field across the southern part of the belt [Bennett et al., 1999]. However, structural correlations, paleocurrent data [Snow and Wernicke, 1989; Snow et al., 1993; Snow and Prave, 1994], and some paleomagnetic data (J. K. Snow and J. W. Geissman, unpublished data, 1995), which document modest to significant counterclockwise rotations, are inconsistent with regionally pervasive dextral shear. These opposite-sense rotations have instead been interpreted as an expression of north-south shortening in an overall constrictional strain field [Wernicke et al., 1988b; Snow and Prave, 1994].

[4] Recent debate has also focused on how Neogene crustal deformation is partitioned between strike-slip and normal fault modes of extension in the Death Valley region. For example, a strain field dominated by map-view, domino-style rotation of range blocks [Hornafius et al., 1986] has been proposed [Serpa and
Pavlis, 1996] on the basis, principally, of reinterpreted paleomagnetic data from crystalline rocks in the Black Mountains of central Death Valley (Figure 1) [Holm et al., 1993]. This emphasis on distributed shear and block rotation differs significantly from earlier hypotheses that emphasize crustal extension dominated by normal faulting [Wright and Troxel, 1973; Stewart, 1983; Wernicke et al., 1988b; Topping, 1993].

[5] To evaluate these contrasting hypotheses, we quantify magnitudes of vertical axis rotation using paleomagnetic data, collected along a transect of the Death Valley region roughly parallel to $36^{\circ} \mathrm{N}$ (Figure 1). Paleomagnetic sampling sites were chosen in Tertiary shallow level intrusive or extrusive rocks and Paleozoic carbonate strata. The igneous rocks are well dated and span most or all of the extensional history of the region.

\section{Tectonic Setting}

[6] The Death Valley area (Figure 1) is probably the most strongly extended region in the U.S. Cordillera. This region has long been considered an ideal natural laboratory for assessing models of 
intracontinental extension [e.g., Wernicke et al., 1988b], extensional basin formation [e.g., Snow and Lux, 1999; Wright et al., 1999], and the role of strike-slip faulting associated with large-scale extension [e.g., Serpa and Pavlis, 1996]. Deformation in the Death Valley extended terrane is accommodated by west dipping normal faults and both right- and left-lateral strike-slip faults, active principally in Neogene time [Wernicke et al., 1988b]. The strike-slip faults appear to be coeval with normal faulting and to have left-lateral sense when they strike eastward or northeastward (e.g., Garlock fault) and rightlateral sense when they strike northwestward (e.g., northern Death Valley-Furnace Creek fault zone [Wright, 1976]). Thus the conjugate strike-slip fault systems also accommodate relative westward motion of the Sierra Nevada away from the Spring Mountains.

[7] Although extension may have begun as early as Oligocene time in the Death Valley region [Cemen et al., 1985; Snow and Lux, 1999], the rate of extension appears to have peaked between about $12 \mathrm{Ma}$ and the present [Wright et al., 1983, 1984; Holm and Dokka, 1991; Holm et al., 1992; McKenna and Hodges, 1989; McKenna and Snee, 1990]. The locus of extension progressed westward with time [Wright et al., 1983, 1984; Wernicke, 1992], such that most extension east of Death Valley is pre-Pliocene, whereas major Pliocene and Quaternary extension characterizes Death Valley [Knott et al., 1999] and ranges to the west.

\subsection{Greenwater Range}

[8] The Greenwater Range consists mostly of variably faulted and tilted 10.5 to $\sim 4$ Ma volcanic rocks [Haefner, 1974; Wright et al., 1991; Thompson et al., 1993]. The volcanic rocks include 10.59.5 Ma calc-alkalic dacite/rhyolite flows, the 8.5-7.5 Ma Shoshone Volcanics, and the 7-5 Ma Greenwater Volcanics [Fleck, 1970; Wright et al., 1991]. These rocks are locally overlain by subhorizontal basalt flows and conglomerates of the 5-4 Ma Funeral Formation. The Shoshone Volcanics rest nonconformably on the Chocolate Sundae Mountain granite, which yielded a biotite ${ }^{40} \mathrm{Ar} /{ }^{39} \mathrm{Ar}$ plateau date of $9.75 \pm 0.2 \mathrm{Ma}$ [Holm et al., 1992] and two zircon fission track dates of $8.7 \pm 1.2 \mathrm{Ma}$ and $7.5 \pm 0.8 \mathrm{Ma}$ [Holm and Dokka, 1993]. Contact relations and age data indicate that the Chocolate Sundae Mountain granite was unroofed and exposed by 9.5 to $\sim 8.0 \mathrm{Ma}$ [Holm et al., 1994a]. On the basis of the orientation of the overlying volcanic rocks, the Chocolate Sundae Mountain granite has been tilted a minimum of $30^{\circ}$ eastward since emplacement, although it is probable that some tilting preceded the eruption of these rocks. A decreasing east dip (growth character) in younger volcanic rocks indicates that $\sim 15^{\circ}$ of the east tilting occurred before $7 \mathrm{Ma}$.

\subsection{Black Mountains}

[9] The Black Mountains (Figure 1) are predominantly composed of crystalline rocks that form the deeper parts of a tilted (west-side-up) crustal section [Holm and Wernicke, 1990; Holm et al., 1993; Holm and Dokka, 1993; Topping, 1993; Mancktelow and Pavlis, 1994]. The crystalline complex in the western part of the range is exposed in three NW trending antiforms known as the Death Valley "turtlebacks." The complex is crudely stratiform and includes from structural bottom to top Proterozoic gneisses, nonconformably overlying Neoproterozoic strata, and sheet-like middle to late Miocene mafic to silicic plutons. Although older fabrics are locally preserved [Miller and Friedman, 1999], amphibolite facies metamorphism and the development of a pervasive LS tectonite, with NW trending lineation, occurred mainly in Miocene time [Holm et al., 1992; Mancktelow and Pavlis, 1994; Miller, 1999]. Thermochronologic and barometric studies indicate that the crystalline core was rapidly exhumed from midcrustal depths between 6 and 9 Ma [Holm et al., 1992; Holm and Dokka, 1993]. The unroofing was therefore coeval with major volcanism in the adjacent Greenwater Range [Thompson et al., 1993].
[10] In the western part of the range (Figure 1), an 11.7 Ma mafic pluton is locally ductilely deformed and folded along with Proterozoic country rocks [Asmerom et al., 1990; Holm et al., 1994b; Mancktelow and Pavlis, 1994]. It is intruded by undeformed silicic plutons and mafic to silicic dikes ranging in age from $\sim 9$ to $6.5 \mathrm{Ma}$ [Holm et al., 1992; Holm, 1995]. Paleomagnetic results from these younger intrusions generally yield high laboratory unblocking temperature, high-coercivity magnetizations with inclinations similar to expected Miocene values [Holm et al., 1993]. Magnetizations have discordant declinations, however, that were interpreted to suggest that large-magnitude $\left(50^{\circ}-80^{\circ}\right)$ clockwise rotation of the crystalline core occurred late in its deformational history, presumably in response to postunroofing dextral shear along the Death Valley fault zone [Holm et al., 1993]. Megascopically undeformed parts of the older mafic batholith yield a magnetite-dominated remanent magnetization of high coercivity and high laboratory unblocking temperatures that is interpreted to predate unroofing from midcrustal depths [Holm et al., 1993].

\subsection{Panamint Mountains}

[11] To first order, the Panamint Mountains are also an east tilted crustal section, with an antiformal axis near the crest of the range interpreted as the result of extensional "rollover" in the Miocene [Hodges et al., 1987; Wernicke et al., 1988a]. In contrast to the Black Mountains the range includes predominantly miogeoclinal strata without significant Tertiary dynamic metamorphism [Labotka and Albee, 1988]. Along the east flank of the rollover, the miogeoclinal strata are unconformably overlain by moderately to steeply east tilted Tertiary strata. On the western flank, strata near the base of the miogeocline are metamorphosed and ductilely deformed. The Panamints are similar to other ranges in the area (e.g., Funeral, Cottonwood, and Nopah-Resting Spring Ranges) in that the miogeoclinal assemblage contains east directed thrust faults that serve as markers for reconstructing Neogene deformation in the region [Snow and Wernicke, 1989, 2000]. These reconstructions support Stewart's [1983] interpretation that the range was detached from above the crystalline core of the Black Mountains and translated some $80 \mathrm{~km}$ northwest along one or more west dipping low-angle detachment faults.

[12] The structure of the central part of the range near Telescope Peak, where the majority of our paleomagnetic sampling was conducted, is a gently dipping homocline, of Neoproterozoic strata near and east of the crest of the antiform, which is locally steeply tilted and cut by normal faults syntectonic with the intrusion of the $\sim 10.6$ Ma Little Chief stock. Emplacement of the stock appears to have occurred prior to most of the eastward tilting of the range because volcanic strata dated at $\sim 10.5 \mathrm{Ma}$ are nearly as steeply tilted as underlying Paleozoic strata [McKenna and Hodges, 1990].

[13] The northern part of the range, at and near Tucki Mountain, where we collected another group of samples, is characterized by steeply tilted, imbricately normal faulted miogeoclinal strata, overlain by Miocene volcanic strata with only gentle discordance. At deep levels, prior to tilting, the section was intruded by the midCretaceous Skidoo muscovite granite and cut by the east vergent Panamint thrust fault [Hunt and Mabey, 1966; Hodges et al., 1987; Wernicke et al., 1988a; Wernicke, 1993].

\section{Methods}

\subsection{Sampling Localities}

[14] This study is based on demagnetization results from 705 independently oriented samples collected from 104 sites in the Greenwater Range and Black and Panamint Mountains (Figure 2) in 1993, 1994, and 1996. Samples from 78 sites were collected using a portable gas-powered drill with nonmagnetic drill bits. Each sample was individually oriented using both solar and magnetic compasses. Oriented block samples were collected for 


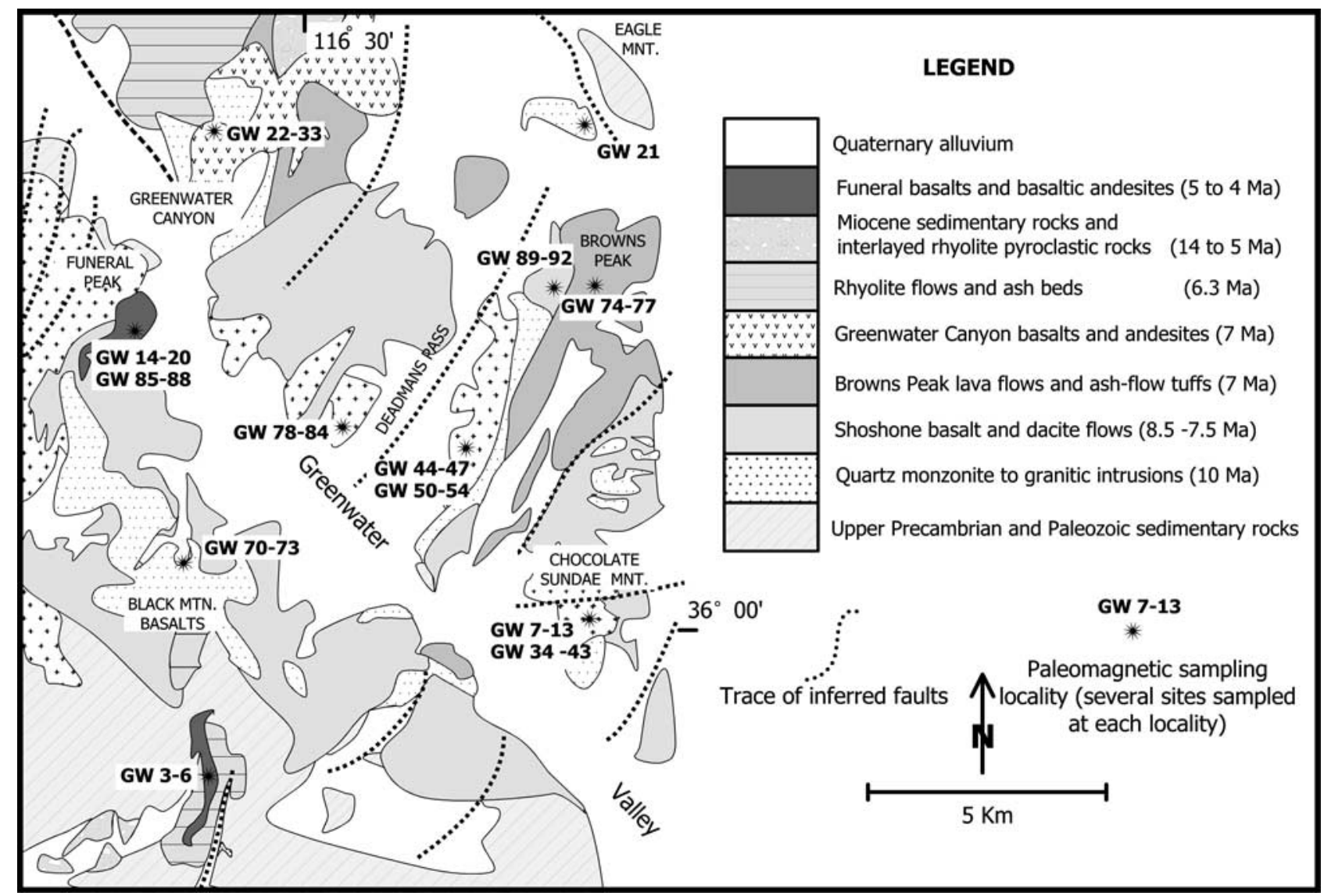

Figure 2. Simplified geologic map showing paleomagnetic sampling locations in the Greenwater Range and eastern part of the Black Mountains (modified after Wright et al. [1991] and R. Thompson (unpublished mapping, 1993)).

26 sites and later drilled with nonmagnetic drill bits in the laboratory. Sample sites consisted of single outcrops or a series of outcrops over a small area $\left(<50 \mathrm{~m}^{2}\right)$.

[15] Upper Miocene basalt, pyroclastic rocks, and mid-Miocene intrusive rocks were sampled in three general areas in the Greenwater Range. The sampling included 13 sites in the 7 to $5 \mathrm{Ma}$ Greenwater Volcanic rocks (sites GW21-GW33), eight sites in the 7 Ma Browns Peak pyroclastic rocks (equivalent to the Shoshone volcanics; sites GW74-GW77 and GW89-GW92), 14 sites in the 9.76 Ma Chocolate Sundae Mountain pluton (sites GW7-GW13, GW34-GW36, GW39-GW41, and GW43), and 21 sites in intrusive rocks that crop out north and south of Deadmans Pass (sites GW44-GW47, GW50-GW54, GW56-GW60, and GW78-GW84).

[16] Upper Miocene basalt flows were sampled at two localities in the Black Mountains. These include four sites in the 8.5 to 7.5 Ma Black Mountain basalts (sites GW70-GW73) and 15 sites in the Funeral Peak basalts (sites GW3-GW6, GW14-GW20, and GW85-GW88). Mid-Miocene volcanic and intrusive rocks and Paleozoic carbonate rocks were sampled at three localities in the Panamint Mountains. These include nine sites in the mid-Miocene(?) Dinosaur Ridge basalts (sites TB1-TB9), 11 sites in the 10.6 Ma Little Chief stock (sites LC1-LC9, TD1, and ZJ1), and eight sites in Paleozoic carbonate strata (sites OP1-OP8). For all localities involving volcanic rocks we sampled every wellexposed, separate cooling unit possible.

\subsection{Analytical Methods}

[17] Each core was cut into $2.5 \mathrm{~cm}$ by $2.5 \mathrm{~cm}$ cylindrical specimens, using a nonmagnetic saw blade. Remanent magnet- izations were measured using a 2G Enterprises Model 760R threeaxis superconducting rock magnetometer with an integrated alternating field (AF) demagnetization system at the University of New Mexico Paleomagnetism Laboratory. Specimens were progressively AF demagnetized in, typically, $15-20$ steps to a maximum field of $120 \mathrm{mT}$. Samples with high coercivity were treated with thermal demagnetization using a Schonstedt TSD-1 furnace up to maximum temperatures of $615-680^{\circ} \mathrm{C}$, depending on rock type and behavior. Typically, $8-10$ specimens were demagnetized per site, one specimen per sample unless additional thermal treatment was performed to verify the reliability of the alternating field results.

[18] Demagnetization results were plotted on modified orthogonal demagnetization diagrams [Roy and Park, 1974], and linear segments through selected demagnetization data points were calculated using principal component analysis [Kirschvink, 1980]. Site-mean directions were calculated following the methods of Fisher [1953]. For most samples an unanchored, best fit line segment was fit to the demagnetization data points. Best fit magnetization vectors, in most cases, involved $10-15$ data points, but as few as 3 to as many as 25 were used. Specimen maximum angular deviation (MAD) values for linear data, typically, were $<4^{\circ}$ but ranged from $<1^{\circ}$ to as high as $15^{\circ}$. Magnetization directions were (rarely) anchored to the origin when the difference between the anchored and unanchored directions was $<10^{\circ}$ and resulted in an improved MAD value. To objectively consider populations with some anomalous results, sample magnetization directions were considered outliers and rejected from the site-mean calculation if the angular distance between the sample direction and the initial estimated site mean was $>15^{\circ}$. A single-component magnetization that decayed to the origin was isolated in most samples, although 
Table 1. Paleomagnetic Data From the Death Valley Extended Terrane ${ }^{\mathrm{a}}$

\begin{tabular}{|c|c|c|c|c|c|c|c|c|c|c|c|c|}
\hline Rock & Site & $R$ & $N / N_{0}$ & $\alpha_{95}$ & $\kappa$ & $D_{\text {in situ }}$ & $I_{\text {in situ }}$ & $\mathrm{St} / \mathrm{Dp}$ & $D_{\text {cor }}^{\mathrm{e}}$ & $I_{\text {cor }}{ }^{\mathrm{e}}$ & $\begin{array}{c}\text { Latitude, } \\
{ }^{\circ} \mathrm{N}\end{array}$ & $\begin{array}{c}\text { Longtitude, } \\
{ }^{\circ} \mathrm{W}\end{array}$ \\
\hline \multicolumn{13}{|c|}{ Greenwater Range: Greenwater Canyon (7-5 Ma) } \\
\hline Basalt flow & GW21 & 7.90 & $8 / 9$ & 6.7 & 68.5 & 320.9 & 36.9 & & 320.9 & 36.9 & 36.37 & 116.50 \\
\hline Basalt flow & GW22 & 7.98 & $8 / 9$ & 2.7 & 438.2 & 4.9 & 40.4 & $175 / 06$ & 359.8 & 41.2 & 36.18 & 116.55 \\
\hline Basalt flow & GW23 & 3.99 & $4 / 9$ & 4.9 & 353.5 & 345.4 & 44.6 & $175 / 06$ & 339.7 & 43.3 & 36.18 & 116.55 \\
\hline Basalt flow & GW24 & 6.96 & $7 / 8$ & 5.2 & 136.3 & 175.9 & -32.6 & $035 / 29$ & 197.7 & -46.8 & 36.18 & 116.55 \\
\hline Basalt flow & GW25 & 9.74 & $10 / 11$ & 8.3 & 34.6 & 185.6 & -31.6 & 048/09 & 190.3 & -37.4 & 36.18 & 116.55 \\
\hline Basalt flow & $\mathrm{GW} 27^{\mathrm{b}}$ & 7.96 & $8 / 9$ & 4.4 & 162.9 & 174.3 & -18.4 & $297 / 21$ & 174.9 & 0.1 & 36.18 & 116.40 \\
\hline Tuff & GW28 & 4.93 & $5 / 8$ & 10.3 & 55.8 & 187.1 & -66.2 & $297 / 21$ & 195.6 & -45.9 & 36.20 & 116.40 \\
\hline Tuff & GW29 & 7.87 & $8 / 11$ & 7.7 & 53.4 & 182.3 & -62.3 & $300 / 23$ & 193.4 & -40.9 & 36.20 & 116.40 \\
\hline Tuff & GW30 & 6.80 & $7 / 11$ & 11.1 & 30.5 & 286.8 & 68.1 & $300 / 23$ & 338.0 & 62.5 & 36.20 & 116.40 \\
\hline AFT & GW31 & 9.89 & $10 / 12$ & 5.4 & 80.9 & 342.3 & 68.8 & $300 / 23$ & 5.6 & 49.7 & 36.21 & 116.40 \\
\hline AFT & GW32 & 5.91 & $6 / 10$ & 9.3 & 52.5 & 306.2 & 69.1 & $300 / 23$ & 348.5 & 57.7 & 36.21 & 116.40 \\
\hline Vitrophyre & GW33 & 11.90 & $12 / 12$ & 4.2 & 105.7 & 174.5 & -60.9 & $300 / 23$ & 188.2 & -40.5 & 36.23 & 116.40 \\
\hline Tuff & $\mathrm{GW} 74^{\mathrm{b}}$ & 3.81 & $4 / 7$ & 23.9 & 15.7 & 253.0 & -41.0 & & $\cdots$ & $\ldots$ & 35.90 & 116.60 \\
\hline Mafic dike & GW75 & 10.94 & $11 / 12$ & 3.6 & 164.4 & 350.7 & 41.4 & 030/90 & 350.7 & 41.4 & 35.90 & 116.60 \\
\hline Tuff & GW76 & 7.97 & $8 / 9$ & 3.7 & 222.9 & 185.7 & -43.8 & $160 / 30$ & 153.4 & -49.1 & 35.90 & 116.60 \\
\hline Tuff & GW77 & 9.63 & $10 / 10$ & 10.0 & 24.2 & 178.1 & -31.4 & $085 / 10$ & 178.5 & -41.4 & 35.90 & 116.60 \\
\hline Vitrophyre & GW89 & 8.91 & $9 / 9$ & 5.6 & 86.0 & 353.4 & 30.4 & $025 / 40$ & 23.4 & 42.7 & 35.90 & 116.60 \\
\hline Tuff & $90 \mathrm{a}^{\mathrm{c}}$ & & $4 / 4$ & 7.2 & 162.4 & 205.3 & 1.7 & $050 / 27$ & $\ldots$ & $\ldots$ & 35.90 & 116.60 \\
\hline Tuff & $90 b^{c}$ & & $4 / 4$ & 12.6 & 54.1 & 276.2 & -10.3 & $050 / 27$ & $\ldots$ & $\ldots$ & 35.90 & 116.60 \\
\hline Tuff & $90 c^{b}$ & & $3 / 3$ & 21.6 & 33.6 & 340.4 & 49.5 & $050 / 27$ & $\ldots$ & $\ldots$ & 35.90 & 116.60 \\
\hline Tuff & GW91 & 16.82 & $17 / 17$ & 3.8 & 89.8 & 332.9 & 36.5 & $050 / 27$ & 342.7 & 62.3 & 35.90 & 116.60 \\
\hline Tuff & GW92 & 7.77 & $8 / 10$ & 10.3 & 29.9 & 146.1 & -24.6 & $050 / 27$ & 148.9 & -51.4 & 35.90 & 116.60 \\
\hline \multicolumn{13}{|c|}{ Greenwater Range: Chocolate Sundae Mountain Granite (10-8.5 ma) } \\
\hline Vitrophyre & GW7 & 10.61 & $11 / 12$ & 9.2 & 25.4 & 154.1 & -14.4 & $010 / 30$ & 164.0 & -25.0 & 35.90 & 116.60 \\
\hline Dike & GW9 & 4.96 & $5 / 6$ & 7.3 & 109.8 & 349.1 & 30.1 & $010 / 30$ & 7.0 & 31.0 & 35.90 & 116.60 \\
\hline Monz & GW10 & 8.79 & $9 / 11$ & 8.4 & 38.5 & 303.9 & 18.4 & $010 / 30$ & 315.0 & 42.0 & 35.90 & 116.60 \\
\hline Monz & GW11 ${ }^{b}$ & 3.80 & $4 / 7$ & 24.9 & 14.6 & 271.0 & -21.0 & $010 / 30$ & $\ldots$ & $\ldots$ & 35.90 & 116.60 \\
\hline Dike & GW12 & 6.84 & $7 / 7$ & 10.0 & 37.1 & 155.9 & -44.9 & $010 / 30$ & 118.0 & -50.0 & 35.90 & 116.60 \\
\hline Monzonite & GW35 & 5.68 & $7 / 10$ & 17.5 & 15.5 & 331.1 & 48.2 & $010 / 30$ & 9.0 & 54.0 & 36.00 & 116.50 \\
\hline Monzonite & GW36 & 2.97 & $3 / 6$ & 14.7 & 71.4 & 234.2 & -38.9 & $010 / 30$ & 242.0 & -13.0 & 36.00 & 116.50 \\
\hline Monzonite & GW39 & 3.95 & $4 / 6$ & 11.9 & 60.4 & 348.1 & 49.5 & $010 / 30$ & 23.0 & 47.0 & 36.00 & 116.50 \\
\hline Monzonite & $\mathrm{GW} 40^{\mathrm{b}}$ & 2.63 & $3 / 4$ & 20.6 & 36.9 & 327.7 & 30.7 & $010 / 30$ & $\ldots$ & $\ldots$ & 36.00 & 116.50 \\
\hline Monzonite & $41 / 42$ & 4.77 & $5 / 8$ & 15.4 & 25.5 & 325.5 & 38.8 & $010 / 30$ & 354.0 & 50.0 & 36.00 & 116.50 \\
\hline Monzonite & $\mathrm{GW}_{4} 3^{\mathrm{b}}$ & 1.97 & $2 / 3$ & 43.7 & 34.7 & 204.7 & 43.7 & $010 / 30$ & $\ldots$ & $\ldots$ & 36.00 & 116.55 \\
\hline \multicolumn{13}{|c|}{ Greenwater Range: Deadmans Pass Instrusions, Southern Dedmans Pass ${ }^{\mathrm{d}}$} \\
\hline Monzonite & GW47 & 3.81 & $4 / 6$ & 23.7 & 15.9 & 327.5 & 30.3 & $\mathrm{n} / \mathrm{a}$ & $\ldots$ & $\ldots$ & 36.10 & 116.55 \\
\hline Monzonite & GW50 & 2.99 & $3 / 4$ & 10.4 & 142.2 & 176.1 & -56.8 & $\mathrm{n} / \mathrm{a}$ & $\ldots$ & $\ldots$ & 36.10 & 116.55 \\
\hline Monzonite & GW51 & 2.80 & $3 / 4$ & 21.2 & 19.7 & 355.0 & 50.8 & $\mathrm{n} / \mathrm{a}$ & $\ldots$ & $\ldots$ & 36.10 & 116.55 \\
\hline Monzonite & GW44, 45, & & & & & & & $\mathrm{n} / \mathrm{a}$ & & & 36.10 & 116.55 \\
\hline \multicolumn{13}{|c|}{ Greenwater Range: Deadmans Pass Instrusions, Northern Dedmans Pass ${ }^{\mathrm{d}}$} \\
\hline Monzonite & GW56 $6^{\mathrm{b}}$ & & & & & & & $\mathrm{n} / \mathrm{a}$ & & & 36.10 & 116.55 \\
\hline Monzonite & GW57 & 3.97 & $6 / 8$ & 9.5 & 94.1 & 176.4 & 8.6 & $\mathrm{n} / \mathrm{a}$ & $\ldots$ & $\ldots$ & 36.10 & 116.55 \\
\hline Monzonite & GW58 $8^{\mathrm{b}}$ & & & & & & & $\mathrm{n} / \mathrm{a}$ & $\ldots$ & $\ldots$ & 36.10 & 116.55 \\
\hline Monzonite & GW59 & 2.89 & $3 / 4$ & 29.2 & 18.8 & 31.1 & 62.4 & $\mathrm{n} / \mathrm{a}$ & $\ldots$ & $\ldots$ & 36.10 & 116.55 \\
\hline Monzonite & GW60 & 2.13 & $4 / 7$ & 120.3 & 1.6 & 5.3 & -30.5 & $\mathrm{n} / \mathrm{a}$ & $\ldots$ & $\ldots$ & 36.10 & 116.55 \\
\hline Monzonite & $\begin{array}{c}\text { GW78-84 } \\
46,52,53 \\
54^{\mathrm{d}}\end{array}$ & & & & & & & $\mathrm{n} / \mathrm{a}$ & & & 36.10 & 116.55 \\
\hline \multicolumn{13}{|c|}{ Black Mountains: Funeral Peak Basalts (5-4 Ma) } \\
\hline Basalt flow & GW3 & 7.97 & $8 / 9$ & 3.6 & 237.2 & 161.8 & -35.0 & $027 / 27$ & 183.0 & -50.8 & 35.90 & 116.60 \\
\hline Basalt flow & GW4 & 7.98 & $8 / 8$ & 3.3 & 288.4 & 336.3 & 27.5 & $027 / 27$ & 351.4 & 46.3 & 35.90 & 116.60 \\
\hline Basalt flow & GW5 & 6.97 & $7 / 7$ & 4.3 & 195.1 & 154.0 & -33.4 & $027 / 27$ & 172.6 & -52.5 & 35.90 & 116.60 \\
\hline Basalt flow & GW6 & 6.96 & $7 / 8$ & 4.9 & 150.4 & 153.7 & -33.3 & $033 / 25$ & 168.5 & -53.3 & 35.90 & 116.60 \\
\hline Basalt flow & GW14 & 6.98 & $7 / 8$ & 3.2 & 355.2 & 349.1 & 54.6 & $035 / 15$ & 9.7 & 63.5 & 36.12 & 116.60 \\
\hline Basalt flow & GW15 & 8.00 & $8 / 8$ & 1.3 & 1742.8 & 355.5 & 58.3 & $035 / 15$ & 20.8 & 65.3 & 36.12 & 116.60 \\
\hline Basalt flow & GW16 & 9.96 & $10 / 10$ & 3.4 & 208.9 & 346.8 & 57.5 & $035 / 15$ & 9.8 & 66.7 & 36.12 & 116.60 \\
\hline Basalt flow & GW17 & 9.96 & $10 / 10$ & 3.2 & 225.9 & 350.3 & 57.1 & $035 / 15$ & 13.5 & 65.6 & 36.12 & 116.60 \\
\hline Basalt flow & GW18 & 7.97 & $8 / 8$ & 3.5 & 247.6 & 347.6 & 56.6 & $035 / 15$ & 9.8 & 65.7 & 36.12 & 116.60 \\
\hline
\end{tabular}


Table 1. (continued)

\begin{tabular}{|c|c|c|c|c|c|c|c|c|c|c|c|c|c|c|c|}
\hline \multicolumn{2}{|c|}{ Rock } & Site & $R$ & & $N / N_{0}$ & $\alpha_{95}$ & $\kappa$ & & in situ & $I_{\text {in situ }}$ & $\mathrm{St} / \mathrm{Dp}$ & $D_{\text {cor }}^{\mathrm{e}}$ & $I_{\text {cor }}^{\mathrm{e}}$ & $\begin{array}{l}\text { Latitude, } \\
{ }^{\circ} \mathrm{N}\end{array}$ & $\begin{array}{c}\text { Longtitude, } \\
{ }^{\circ} \mathrm{W}\end{array}$ \\
\hline \multirow{2}{*}{\multicolumn{2}{|c|}{$\begin{array}{l}\text { Basalt flow } \\
\text { Basalt flow }\end{array}$}} & GW87 & 4.95 & & $5 / 5$ & 4.6 & 272.5 & & 57.8 & 57.1 & $035 / 15$ & 22.0 & 63.6 & 36.12 & 116.60 \\
\hline & & GW88 & 4.95 & & $5 / 8$ & 3.2 & 575.7 & & 67.1 & -55.2 & $035 / 15$ & 187.8 & -64.5 & 36.12 & 116.60 \\
\hline \multicolumn{16}{|c|}{ Black Mountains: Black Mountain Basalts $(8.5-7.5 \mathrm{Ma})$} \\
\hline \multicolumn{2}{|c|}{ Basalt flow } & GW70 & 8.91 & & $9 / 11$ & 5.5 & 89.7 & & 64.0 & -62.8 & $010 / 23$ & 211.6 & -63.8 & 35.90 & 116.60 \\
\hline \multicolumn{2}{|c|}{ Basalt flow } & GW71 & 7.79 & & $8 / 10$ & 9.7 & 33.3 & & 16.3 & 68.2 & $010 / 23$ & 57.3 & 57.0 & 35.90 & 116.60 \\
\hline \multirow{2}{*}{\multicolumn{2}{|c|}{$\begin{array}{l}\text { Basalt flow } \\
\text { Basalt flow }\end{array}$}} & GW72 & 8.93 & & $9 / 10$ & 4.8 & 116.0 & & 13.8 & 62.4 & $010 / 23$ & 49.0 & 53.4 & 35.90 & 116.60 \\
\hline & & GW73 & 8.5 & & $9 / 12$ & 12.5 & 18.0 & & 23.5 & 56.8 & $010 / 23$ & 4.1 & 67.7 & 35.90 & 116.60 \\
\hline \multicolumn{16}{|c|}{ Panamint Mountains: Dinosaur Ridge Basalts (11 Ma) } \\
\hline \multicolumn{2}{|c|}{ Basalt flow } & TB1 & 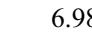 & & $7 / 11$ & 3.8 & 251.4 & & 49.1 & -41.2 & $196 / 39$ & 192.1 & -53.2 & 36.40 & 117.00 \\
\hline \multicolumn{2}{|c|}{ Basalt flow } & TB2 & 7.95 & & $8 / 8$ & 1.9 & 819.3 & & 45.6 & -39.1 & $196 / 39$ & 186.6 & -54.1 & 36 & 117.00 \\
\hline \multicolumn{2}{|c|}{ Basalt flow } & TB3 & 6.93 & & $7 / 10$ & 6.7 & 82.7 & & 48.4 & -38.1 & $196 / 39$ & 187.4 & -52.1 & 36.40 & 117.00 \\
\hline \multicolumn{2}{|c|}{ Basalt flow } & TB4 & 4.95 & & $5 / 5$ & 3.7 & 420.2 & & 88.3 & -38.8 & & 214.3 & -2 & & 117.00 \\
\hline \multicolumn{2}{|c|}{ Basalt flow } & TB5 & 6.95 & & $7 / 9$ & 3.0 & 410.7 & & 91.5 & -45.7 & & 221.5 & -3 & & 117.00 \\
\hline \multicolumn{2}{|c|}{ Basalt flow } & TB6 & 6.97 & & $7 / 9$ & 4.0 & 224.2 & & 69.6 & -46.0 & 196 & 210.6 & -43.0 & 36. & 117.00 \\
\hline \multicolumn{2}{|c|}{ Basalt flow } & TB7 & 7.95 & & $8 / 10$ & 2.5 & 475.7 & & 67.1 & -46.1 & $196 / 39$ & 209.1 & -45.1 & 36.40 & 117.00 \\
\hline \multicolumn{2}{|c|}{ Basalt flow } & TB & 6.95 & & 8/11 & 3.0 & 405.1 & & 67.8 & -46.6 & & 20 & -4 & & 117.00 \\
\hline \multicolumn{2}{|c|}{ Basalt flow } & TB9 & 7.98 & & $8 / 9$ & 2.8 & 400.3 & & 67.7 & -46.6 & $196 / 39$ & 209.7 & -45.6 & 36.40 & 117.00 \\
\hline \multicolumn{16}{|c|}{ Panamint Mountains: Little Chief Stock $(10.5 \mathrm{Ma})$} \\
\hline \multicolumn{2}{|c|}{ Monzonite } & LC7 & 8.9 & & 9/9 & 6.8 & 63.8 & & 60.4 & -50.9 & $358 / 22$ & 188.4 & -5 & 0 & 117.20 \\
\hline \multicolumn{2}{|c|}{ Monzonite } & LC8 & 10.8 & & 11/11 & 13.5 & 12.7 & & 65 & -48.5 & & 19 & -4 & & .20 \\
\hline \multicolumn{2}{|c|}{ Monzonite } & LC9 & 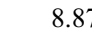 & & 9/9 & 14.9 & 3.1 & & 46.1 & -36.2 & 358 & 146.0 & -50.2 & 36 & 117.20 \\
\hline Monzo & & LC12 & 10.93 & & 11/11 & 4.4 & 109.4 & & 61.8 & -46.6 & $358 / 22$ & 184.8 & -48.6 & 36.20 & 117.20 \\
\hline Monzo & & LC14 & 6.85 & & $7 / 7$ & 15.3 & 13.3 & & 60.2 & -49.8 & $358 / 22$ & 188.2 & -52.8 & & 117.20 \\
\hline Monzo & & $\mathrm{LC} 18^{\mathrm{d}}$ & 8.8 & & $9 / 9$ & 16.6 & 10.7 & & 75.7 & 24.3 & $358 / 22$ & & & & 117.20 \\
\hline Monzo & & LC19 & 11.92 & & $12 / 12$ & 6.3 & 49.3 & & 70.7 & -47.6 & $358 / 22$ & 193.7 & -46.6 & 36 & 117.20 \\
\hline Monzo & & LC20 & 10.87 & & 11/11 & 12.0 & 15.5 & & 58.3 & -60.9 & $358 / 22$ & 199.3 & -61.9 & 36.20 & 117.20 \\
\hline Monzo & & $\mathrm{LC} 21^{\mathrm{b}}$ & 8.68 & & $9 / 9$ & 35.9 & 4.8 & & 86.0 & -24.8 & $358 / 22$ & & & & 117.20 \\
\hline Monzo & & LC22 & 4.96 & & $5 / 5$ & 7.7 & 100.6 & & 54.3 & -44.5 & 358 & 177.3 & -49.5 & 36 & 117.20 \\
\hline Mafic & & TD1 & 10.95 & & 11/11 & 5.0 & 51.1 & & 54.4 & -50.7 & $358 / 22$ & 182.4 & -54.7 & 36.20 & 117.20 \\
\hline $\begin{array}{r}\text { Quartzi } \\
\text { conta }\end{array}$ & & ZJ1 & 7.96 & & $8 / 8$ & 5.0 & 133.7 & & 42.1 & -39.2 & $358 / 22$ & 161.1 & -49.2 & 36.20 & 117.20 \\
\hline $\begin{array}{r}\text { Dolom } \\
\text { conta }\end{array}$ & & $\mathrm{ZN} 1^{\mathrm{b}}$ & 9.80 & & 10/10 & 20.3 & 7.3 & & 60.8 & -26.2 & $358 / 22$ & . & & 36.20 & 117.20 \\
\hline Rock & Site & $R$ & $N / N_{0}$ & $\alpha_{95}$ & $\kappa$ & & & $I_{\text {in situ }}$ & $\mathrm{St} / \mathrm{Dp}$ & $D_{\text {co }}$ & $I_{\text {cor }}^{\mathrm{e}}$ & $D_{\text {cor }^{*}}{ }^{\mathrm{e}}$ & $I_{\text {cor }^{*}}{ }^{\mathrm{e}}$ & $\begin{array}{c}\text { Latitude, } \\
{ }^{\circ} \mathrm{N}\end{array}$ & $\begin{array}{c}\text { Longtitude, } \\
{ }^{\circ} \mathrm{W}\end{array}$ \\
\hline & & & & & & & nt 1 & ountains & s: Ordovici & clan Carbo & (ates & & & & \\
\hline lz_carb & OP1 & 5.99 & $6 / 7$ & 2.7 & 600.0 & 310 & & 36.2 & $002 / 108$ & 59.0 & 25.2 & 344.0 & 67.0 & 36.50 & 117.30 \\
\hline $\mathrm{Plz}$ carb & OP2 & 6.33 & $5 / 7$ & 9.7 & 62.2 & 305 . & & 31.1 & $000 / 90$ & 46.2 & 45.1 & 328.0 & 66.0 & 36.50 & 117.20 \\
\hline Plz_carb & OP3 & 6.87 & $6 / 7$ & 8.2 & 67.5 & 34 & & 60.0 & $353 / 37$ & 35.7 & 49.1 & 54.0 & 58.0 & 36.50 & 117.20 \\
\hline Plz_carb & OP4 & 4.81 & $5 / 6$ & 17.3 & 20.5 & 298 & & 19.4 & $354 / 90$ & 26.2 & 52.4 & 307.0 & 57.0 & 36.50 & 117.20 \\
\hline Plz_carb & OP5 & 6.88 & $7 / 8$ & 8.8 & 47.9 & 285 & & 15.8 & $000 / 70$ & 348.4 & 71.8 & 291.0 & 55.0 & 36.40 & 117.10 \\
\hline $\mathrm{Plz}$ carb & OP6 & 10.70 & $10 / 11$ & 4.7 & 104.7 & 312 . & & 25.1 & $003 / 45$ & 344.7 & 53.1 & 335.0 & 57.0 & 36.40 & 117.10 \\
\hline Plz_carb & OP7 & 8.09 & $7 / 10$ & 20.5 & 9.5 & 303. & & 45.7 & $346 / 68$ & 29.1 & 45.7 & 359.0 & 78.0 & 36.30 & 117.10 \\
\hline $\mathrm{Plz}$ carb & OP8 & 3.87 & $4 / 9$ & 19.4 & 23.4 & 42. & & 50.9 & $010 / 54$ & 66.6 & 10.9 & 68.0 & 25.0 & 36.40 & 117.10 \\
\hline
\end{tabular}

${ }^{\text {a }} N / N_{0}$ is ratio of samples used $(N)$ to samples collected $\left(N_{0}\right) ; \alpha_{95}$ is $95 \%$ confidence interval about the estimated mean direction, assuming a circular distribution; $\mathrm{k}$ is the best estimate of (Fisher) precision parameter; $D_{\text {in situ }}$ and $I_{\text {in situ }}$ are in situ declination and inclination, degrees; St/Dp are strike and dip of the site (dip direction $90^{\circ}$ clockwise from strike); $D_{\text {cor }} / I_{\text {cor }}$ are corrected declination and inclination; Lat, latitude; Long, longtitude; n/a indicates not available. AFT is ash fall tuft. Plz carb refers to Paleozoic carbonate rocks.

${ }^{\mathrm{b}}$ Dispersion at site level unacceptably high and therefore rejected from further analysis.

${ }^{\mathrm{c}}$ Multiple demagnetization vectors isolated within samples from the same site, rejected from further analysis.

d All sites rejected; see text discussion.

${ }^{\text {e }}$ Two different tilt corrections werw applied to these units. $D_{\text {cor }}$ and $I_{\text {cor }}$ refer to bedding orientations at each site in Pogonip Group strata; $D_{\text {cor* }}$ and $I_{\text {cor* }}$ refer to a regional tilt correction based on the orientation of the Tertiary basalt section at Dinosaur Ridge $\left(016^{\circ} / 39^{\circ}\right.$, east dip).

some variability in response, discussed in detail in section 4 , was reflected because of the rock type analyzed. Stable endpoint magnetizations are typically well grouped at the site level (Table 1). Sites with dispersed directions, with $\alpha_{95}$ values $>15^{\circ}$ and $/$ or $\kappa$ values $<10$, were rejected from the calculated group-mean direction. Site locations and rock types are shown in Figures 1, 2, and 3.

\section{Paleomagnetic Results}

[19] Paleomagnetic sites are discussed by geographic location in three groups (Greenwater Range, Black Mountains, and Panamint Mountains) and then further subdivided into eight groups based on rock type. Site-mean results for 78 of the 104 sites were calculated from interpretable and internally consistent demagnetization data (Table 1). Twenty-six sites, largely from intrusions in the Greenwater Range, did not yield stable endpoint demagnetization behavior or site-mean directions of reasonable precision $(\kappa$ values $<10)$. Of the 78 accepted sites, 72 sites were used to calculate group-mean magnetization directions (Table 2). The six sites that were excluded from group-mean calculations either had site-mean directions that fell more than two angular standard deviations away from the overall group-mean direction $(n=4)$ or were the only sites from the sampling locality that yielded interpretable results $(n=2)$. 


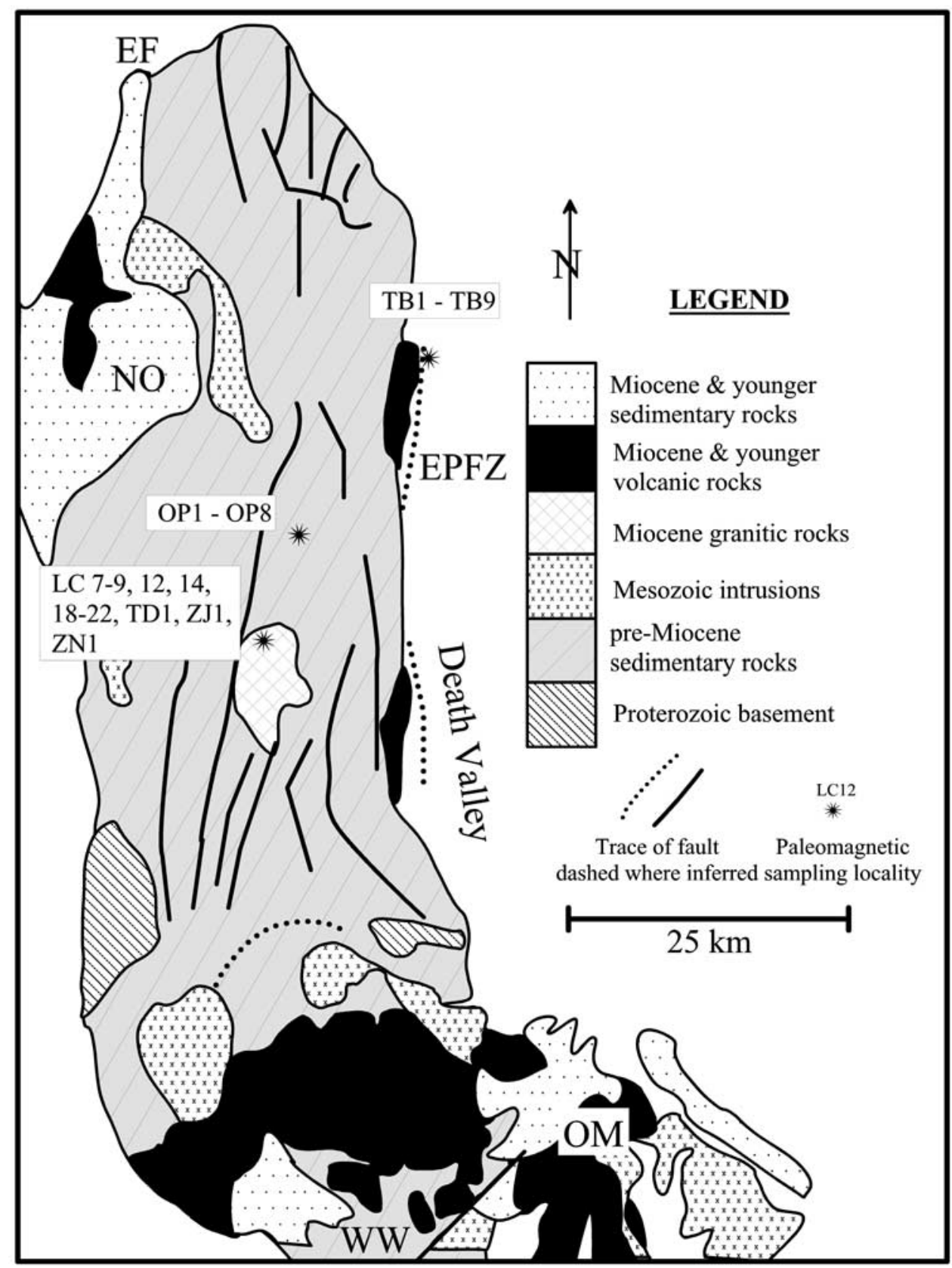

Figure 3. Simplified geologic map of the Panamint Mountains depicting paleomagnetic sampling localities in this study. NO, Nova Basin; EF, Emigrant fault; EPFZ, Eastern Panamint fault zone; WW, Wingate Wash fault zone; OM, Owlshead Mountains. (Modified from Wright et al. [1991].)

[20] In general, most samples contain one magnetization component that is usually well grouped at the site level (Figure 4; compare Figure 4e with Figure 4g). Some samples exhibit more complicated multicomponent demagnetization behavior with $\sim 25 \%$ of the samples exhibiting demagnetization behavior defining two components of magnetization (Figures $4 \mathrm{~g}$ and $4 \mathrm{~h}$ ). Typically, AF demagnetization effectively removed any remanence overprint by $\sim 20 \mathrm{mT}$, with the remaining magnetization decaying linearly to the origin with $<10 \%$ of the natural remanent magnetization (NRM) intensity remaining by $120 \mathrm{mT}$. Multicomponent behavior is generally restricted to some pyroclastic, intrusive, and carbonate rocks. In samples with more than one magnetization component, a viscous remanent magnetization (VRM) or secondary magnetization, of varying relative intensity, is superimposed on the stable endpoint component. For some rock types, the stable endpoint magnetization was not fully isolated by $\mathrm{AF}$ demagnetization; the magnetization did not decay toward the origin and a considerable fraction $(\sim 20-40 \%)$ of the NRM remained after demagnetization to $120 \mathrm{mT}$ (Figure 4a, GW33; Figure 4b, GW76; Figure 4e, GW17; Figure 4f, TB7; Figure 4g, LC12). For these samples, thermal demagnetization was then used to more fully isolate the stable endpoint magnetization. Maximum VRM unblocking temperatures ranged from $250^{\circ} \mathrm{C}$ to $500^{\circ} \mathrm{C}$. More specifically, demagnetization results varied between geographic location and rock type as discussed in sections $4.1-4.3$.

\subsection{Greenwater Range}

4.1.1. Greenwater Canyon volcanic rocks. [21] Sites in the 7-5 Ma Greenwater Canyon volcanic rocks were collected along a 
Table 2. Paleomagnetic Group Mean Magnetizations From Sites in the Central Death Valley Extended Terrane, California

\begin{tabular}{|c|c|c|c|c|c|c|c|c|c|c|c|c|}
\hline \multirow[t]{2}{*}{ Location } & \multirow[t]{2}{*}{$N / N_{0}$} & \multicolumn{2}{|c|}{ Corrected } & \multicolumn{2}{|c|}{ Tilt Correction } & \multirow[t]{2}{*}{$\kappa$} & \multirow[t]{2}{*}{$\alpha_{95}$} & \multirow[t]{2}{*}{$R$} & \multirow[t]{2}{*}{$P$} & \multirow{2}{*}{$\begin{array}{l}\text { Latitude } \\
{ }^{\circ} \mathrm{N}\end{array}$} & \multirow{2}{*}{$\begin{array}{l}\text { Longtitude } \\
{ }^{\circ} \mathrm{W}\end{array}$} & \multirow[t]{2}{*}{$\mathrm{VGP}^{\mathrm{b}}$} \\
\hline & & $D, \operatorname{deg}$ & $I$, deg & $\mathrm{St}$ & $\mathrm{Dp}$ & & & & & & & \\
\hline \multicolumn{13}{|l|}{ Greenwater Range, $n=56$} \\
\hline Greenwater Canyon basalts & $12 / 13$ & 359.2 & 46.7 & $\mathrm{c}$ & & 30.6 & 8.0 & 11.64 & $7 \mathrm{~N}, 5 \mathrm{R}$ & $36^{\circ} 11^{\prime} 35^{\prime \prime}$ & $116^{\circ} 34^{\prime} 09^{\prime \prime}$ & $17.6^{\circ}$ \\
\hline Browns Peak pyroclastic rocks & $5 / 8$ & 343.7 & 49.7 & c & & 46.8 & 11.3 & 4.91 & $2 \mathrm{~N}, 3 \mathrm{R}$ & $36^{\circ} 07^{\prime} 38^{\prime \prime}$ & $116^{\circ} 22^{\prime} 30^{\prime \prime}$ & $14.1^{\circ}$ \\
\hline Choclate Sundae Granite & $9 / 14$ & 358.3 & 49.8 & $010^{\circ}$ & $30^{\circ}$ & 22.8 & 10.3 & 8.61 & $8 \mathrm{~N}, 2 \mathrm{R}$ & $36^{\circ} 00^{\prime} 41^{\prime \prime}$ & $116^{\circ} 22^{\prime} 30^{\prime \prime}$ & - \\
\hline Deadmans Pass & $2 / 21$ & & & & & & & & & & & \\
\hline \multicolumn{13}{|l|}{ Black Mountains, $n=19$} \\
\hline Funeral Peak basalts & $15 / 15$ & 006.3 & 61.2 & $\mathrm{c}$ & & 93.2 & 4.0 & 14.85 & $9 \mathrm{~N}, 6 \mathrm{R}$ & $36^{\circ} 06^{\prime} 32^{\prime \prime}$ & $116^{\circ} 38^{\prime} 19^{\prime \prime}$ & $10.3^{\circ}$ \\
\hline Black Mountain basalts & $4 / 4$ & 039.0 & 61.9 & $010^{\circ}$ & $23^{\circ}$ & 42.2 & 14.3 & 3.93 & $3 \mathrm{~N}, 1 \mathrm{R}$ & $35^{\circ} 56^{\prime} 36^{\prime \prime}$ & $116^{\circ} 35^{\prime} 00^{\prime \prime}$ & $15.0^{\circ}$ \\
\hline \multicolumn{13}{|l|}{ Panamint Mountains, $n=29$} \\
\hline Ordivician carbonate rocks ${ }^{\mathrm{e}}$ & $6 / 8$ & 028.1 & 45.5 & c & & 11.8 & 20.3 & 5.67 & $6 \mathrm{~N}$ & $36^{\circ} 12^{\prime}$ & $117^{\circ} 18^{\prime}$ & - \\
\hline Ordovician carbonate rocks ${ }^{\mathrm{f}}$ & & $6 / 8$ & 322.0 & 64.0 & $\mathrm{c}$ & 15.6 & 17.5 & 5.68 & $6 \mathrm{~N}$ & $36^{\circ} 12^{\prime}$ & $117^{\circ} 18^{\prime}$ & - \\
\hline Dinosaur Ridge Basalts & $9 / 9$ & 205.6 & -44.3 & $006^{\circ}$ & $39^{\circ}$ & 36.1 & 9.3 & 7.81 & $9 \mathrm{R}$ & $36^{\circ} 24^{\prime}$ & $117^{\circ} 00^{\prime}$ & $15.8^{\circ}$ \\
\hline Little Chief Stock & $9 / 12$ & 181.4 & -51.7 & $358^{\circ}$ & $22^{\circ}$ & 251.3 & 6.5 & 8.86 & 9R & $36^{\circ} 12^{\prime}$ & $117^{\circ} 12^{\prime}$ & - \\
\hline
\end{tabular}

\section{a Greenwater Canyon Volcanic Rocks}

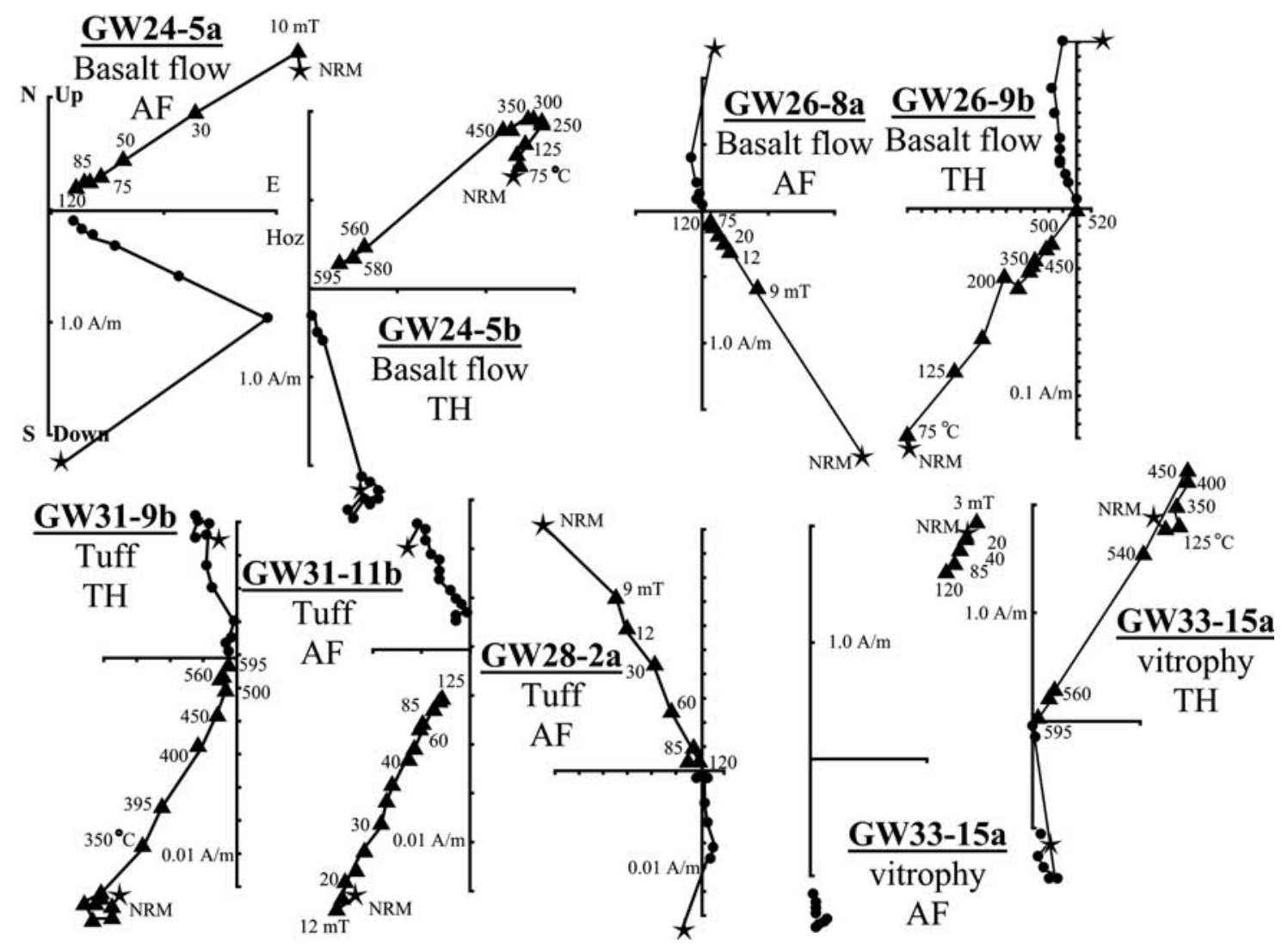

Figure 4. In situ modified orthogonal demagnetization diagrams showing demagnetization response for representative samples collected in the Death Valley region. The NRM is shown as a star in all plots. Solid circles (diamonds) represent the projection onto the horizontal (vertical) plane. In such diagrams the vertical component is plotted versus the true horizontal. AF demagnetization steps are given in millitesla, and thermal demagnetization steps are given in degrees Celsius. Typically, AF and thermal demagnetization results from the specimens of the same sample are shown for comparison. Diagrams are designated with a site location (e.g., GW24), sample number and specimen (-1a), method of treatment (AF or TH), and rock type. NRM intensity in A/m is given for each sample. (a, $\mathrm{b}, \mathrm{c})$ representative $\mathrm{AF}$ and thermal demagnetization diagrams of samples from the Greenwater Range. (d, e) representative AF and thermal demagnetization diagrams of samples from the Black Mountains. (f, g, h) representative AF and thermal demagnetization diagrams of samples from the Panamint Mountains. See text for discussion and Table 1 for rock descriptions. 
b Browns Peak Pyroclastic Rocks
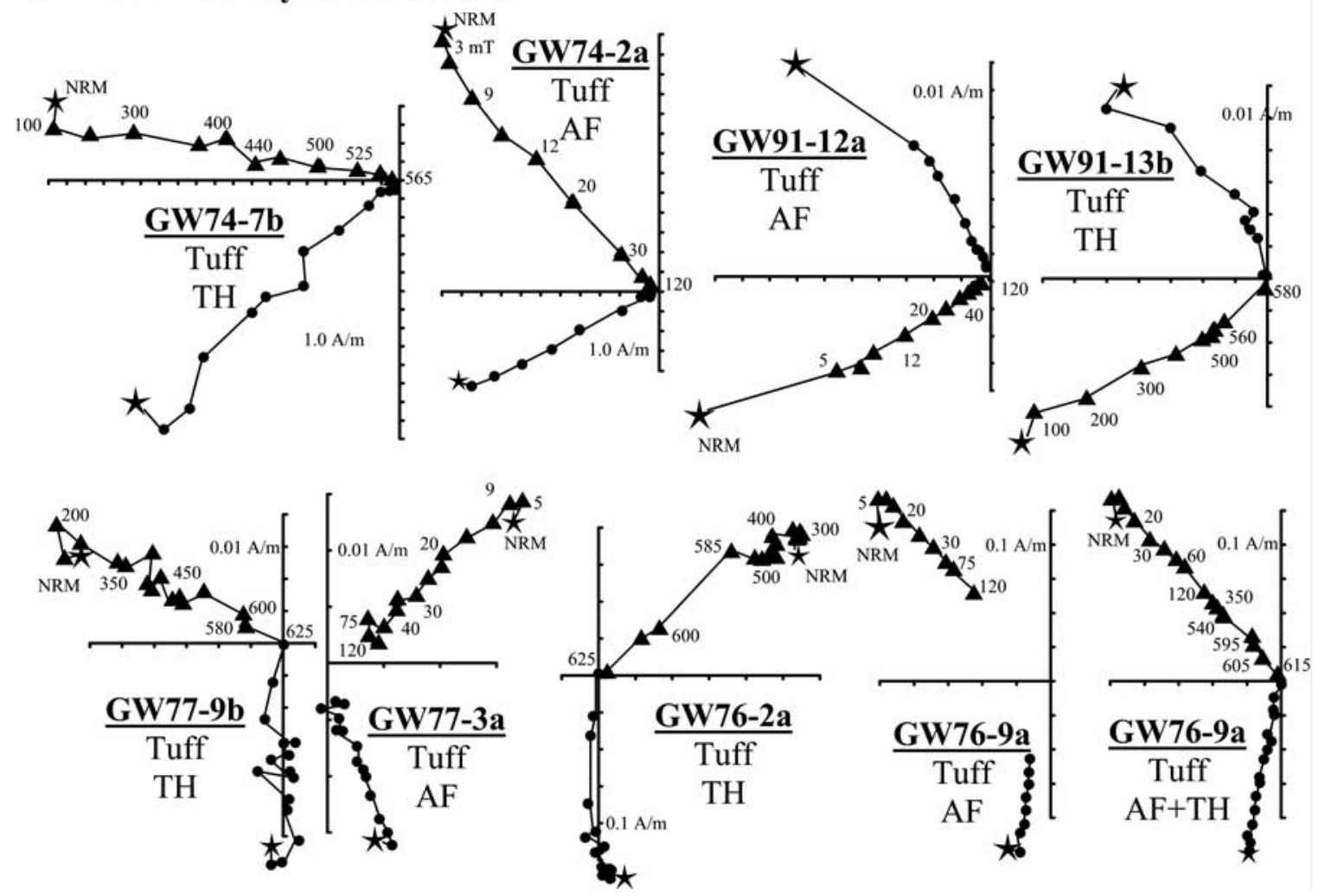

c Chocolate Sundae Mountain Granite
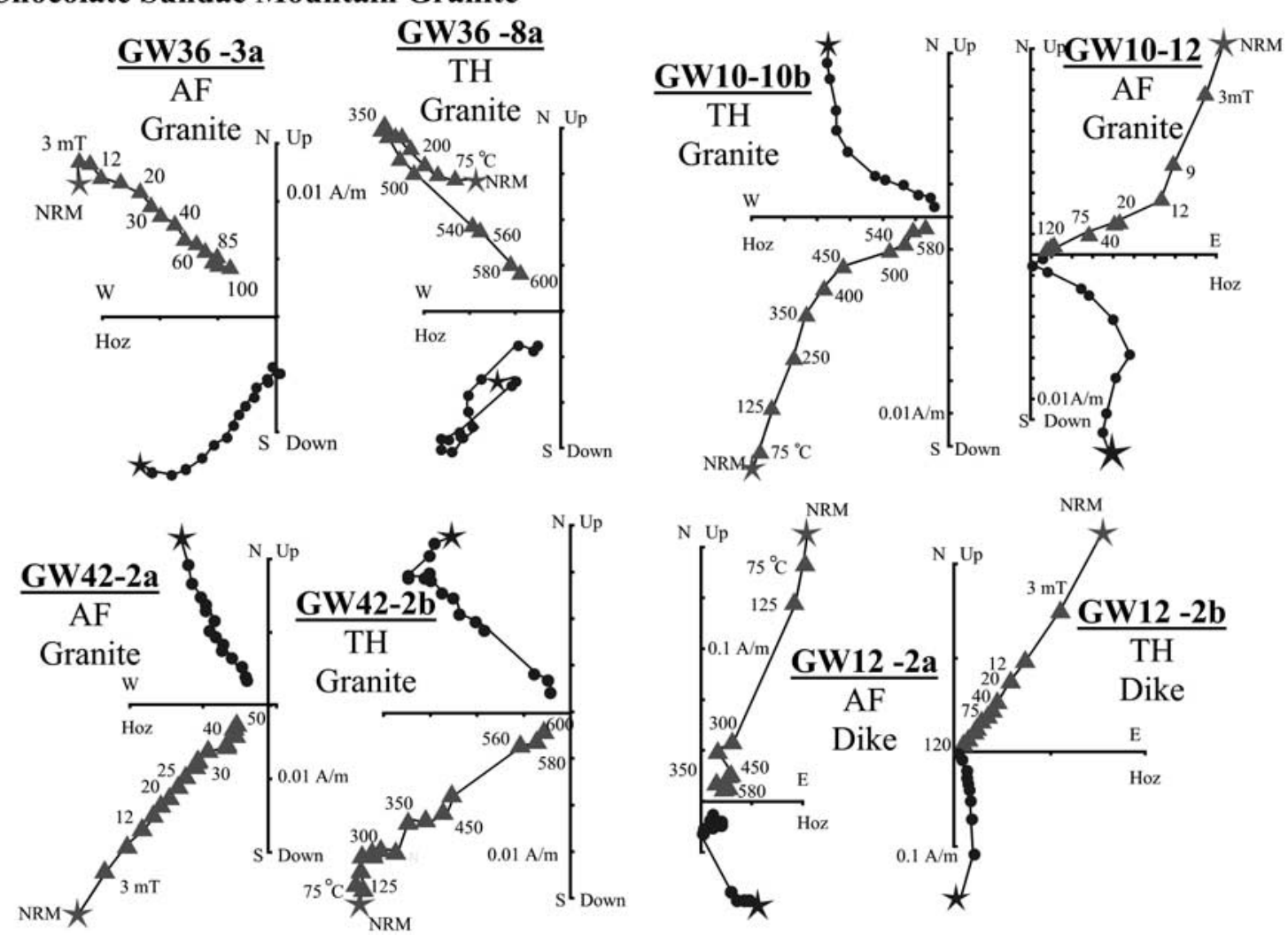

Figure 4. (continued) 


\section{d Black Mountain Basalt Flows}
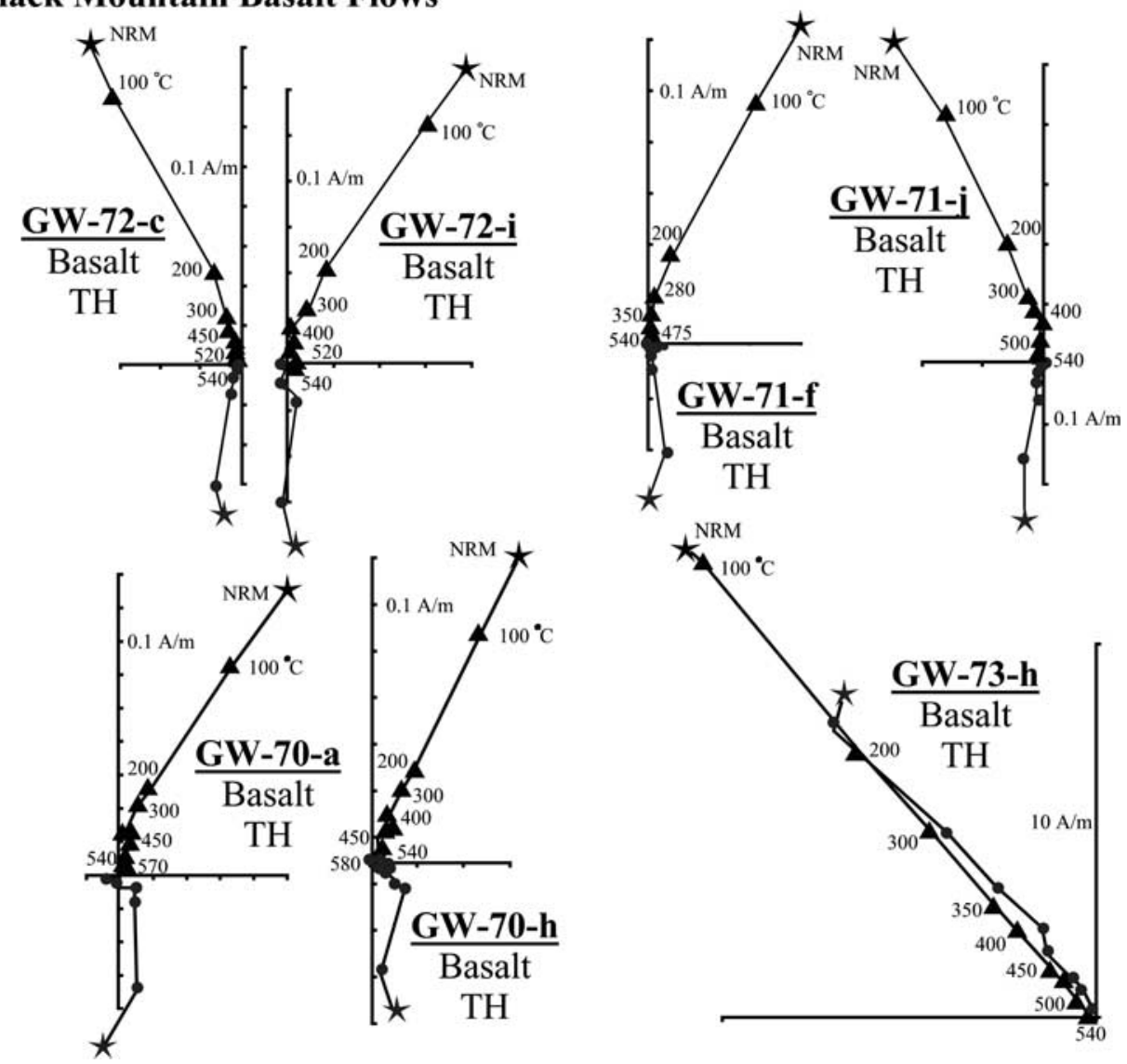

e Funeral Peak Basalt Flows
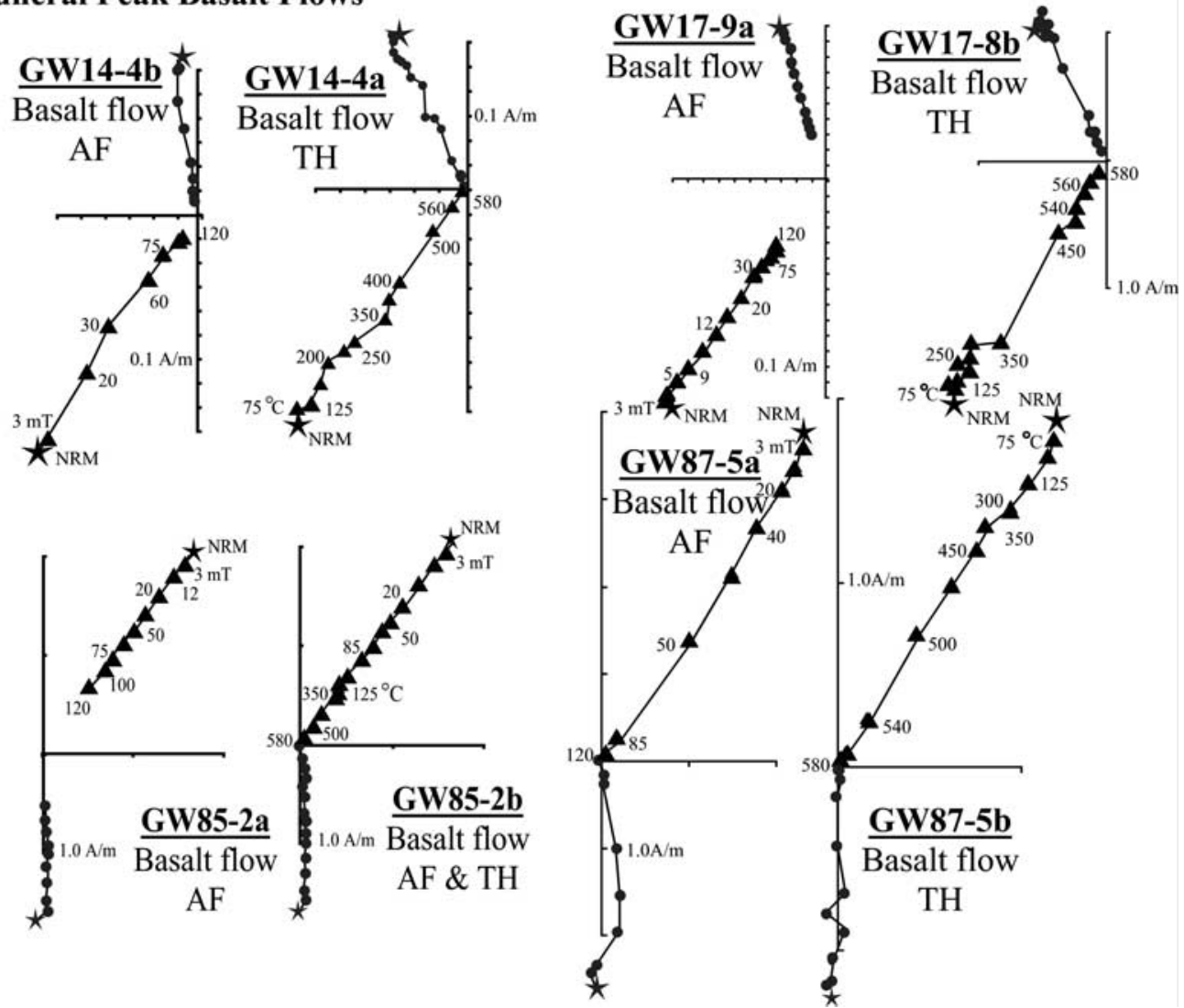

Figure 4. (continued) 


\section{f Dinosaur Ridge Basalt Flows}
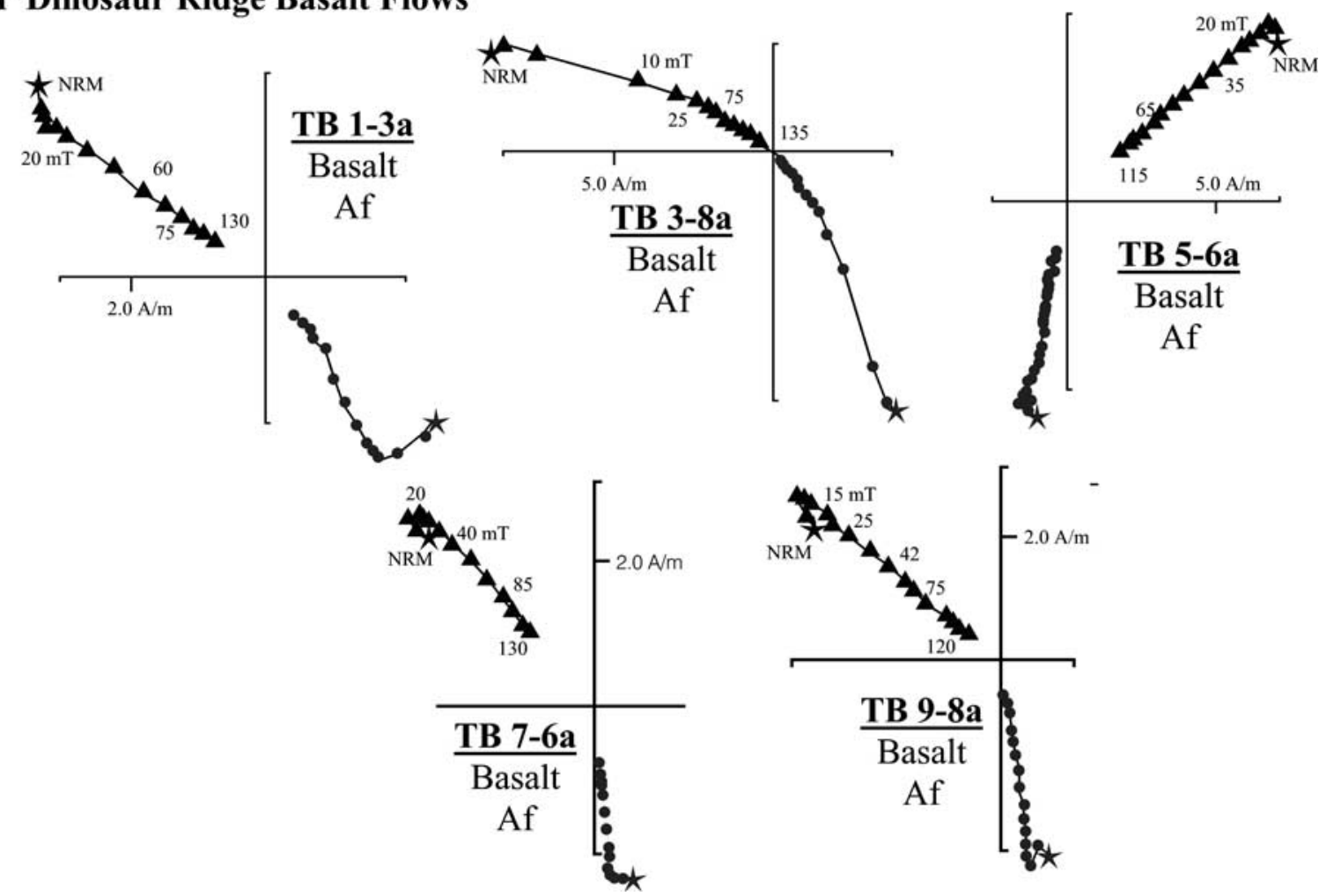

g Little Chief Stock
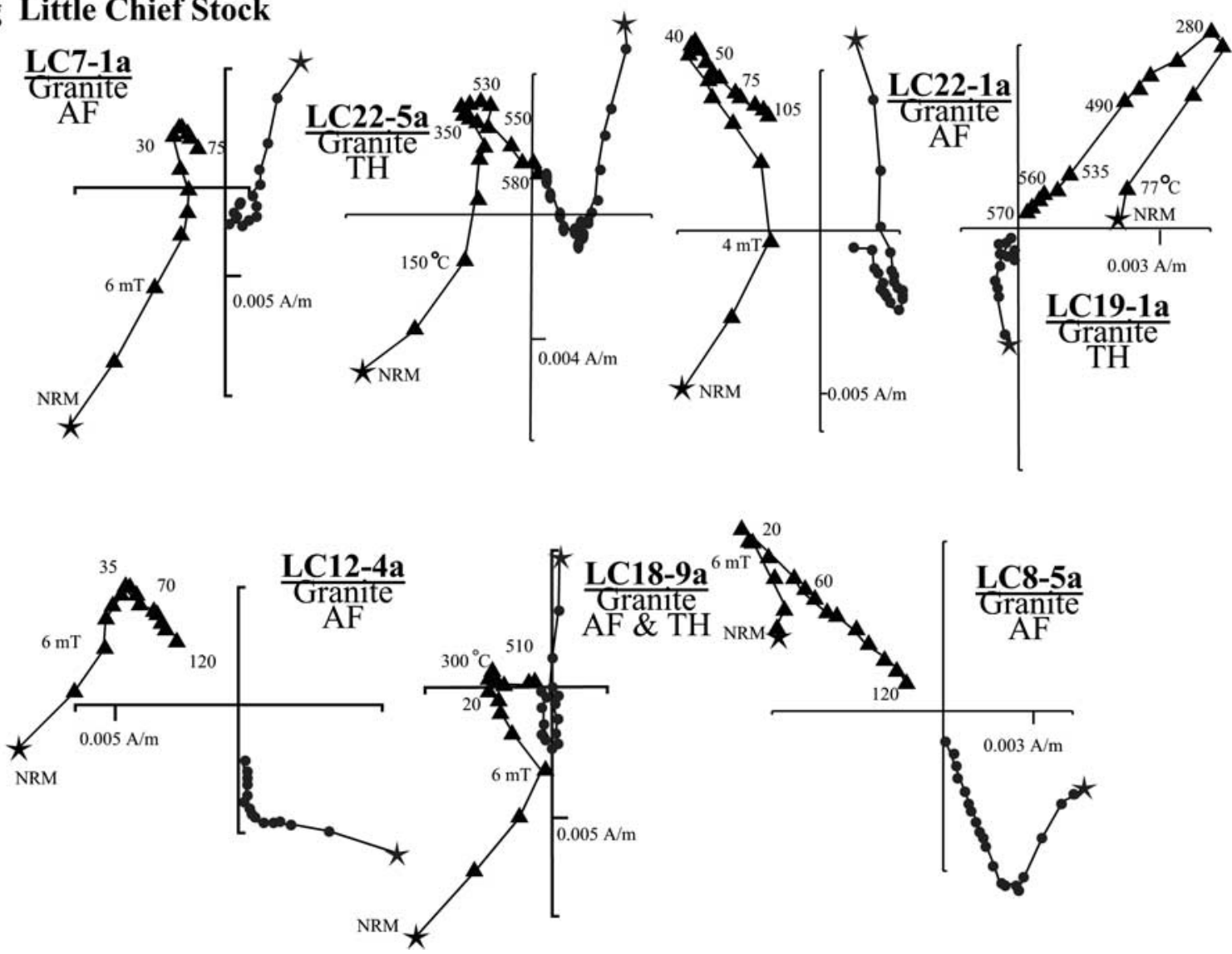

Figure 4. (continued) 
h Pogonip Group Carbonate Rocks
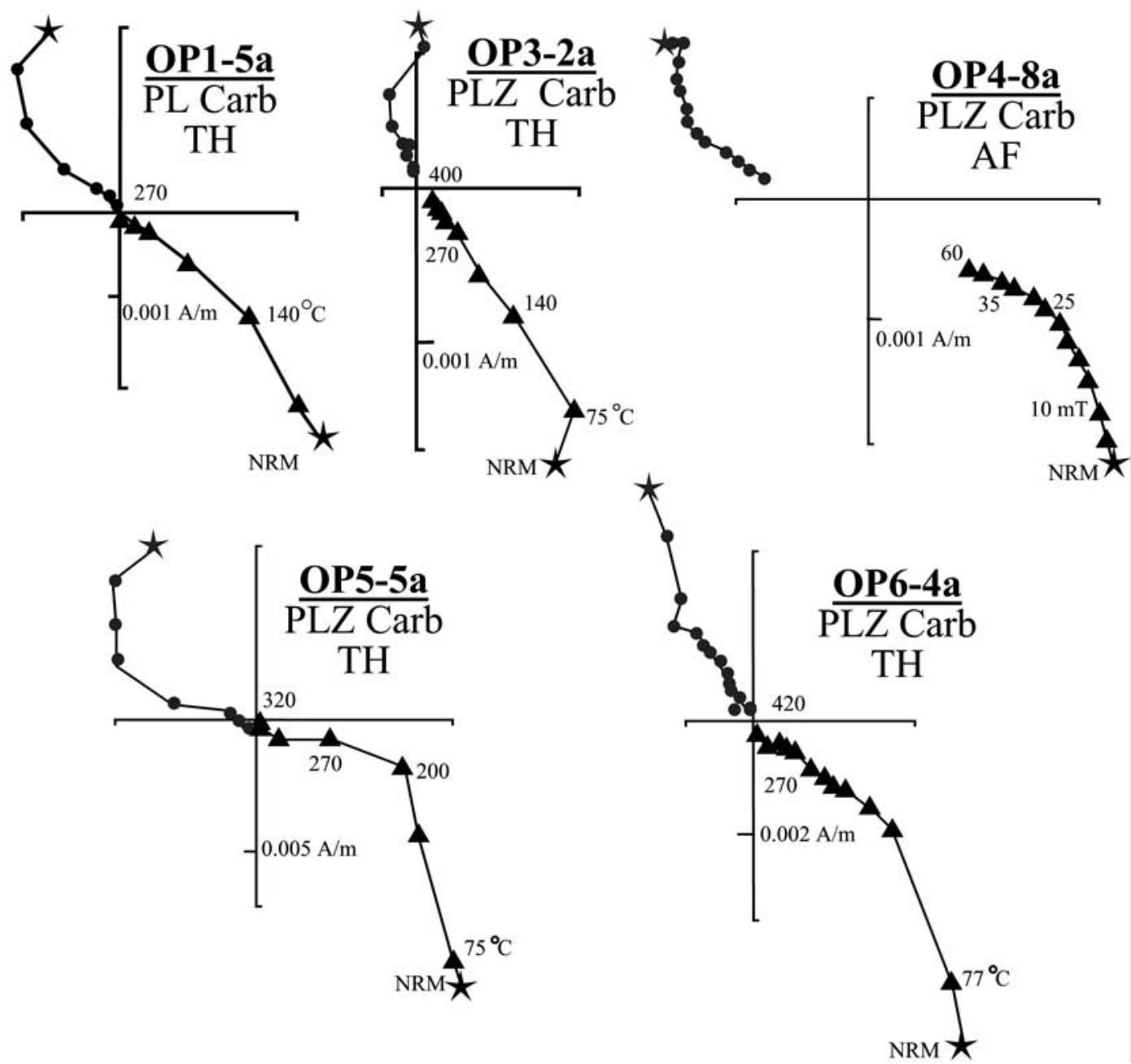

Figure 4. (continued)

transect through Greenwater Canyon, through a gently dipping sequence of basalt flows overlain by pyroclastic rocks (as ash flow cooling units) (Figure 2) [Thompson et al., 1993]. A total of 128 samples were collected from seven sites in basalt flows, one site per flow, and six sites in younger pyroclastic rocks. Generally, the 13 accepted sites yield single-component, wellgrouped magnetizations that are isolated at demagnetization levels above $\sim 15 \mathrm{mT}$ or $250^{\circ} \mathrm{C}$ for basalts and $\sim 20 \mathrm{mT}$ or $350^{\circ} \mathrm{C}$ for pyroclastic rocks (Figure 4a). Basalt flows typically are of high coercivity (median destructive inductions range from 20 to 50 $\mathrm{mT}$ ) and high maximum laboratory unblocking temperatures $\left(520^{\circ}-595^{\circ} \mathrm{C}\right)$. Some sites in pyroclastic rocks required a combination of $\mathrm{AF}$ and thermal demagnetization to isolate the characteristic remanent magnetization (ChRM) (Figure 4a). Overall, pyroclastic rocks are of high coercivity (median destructive inductions range between 40 and $60 \mathrm{mT}$ ) and high laboratory unblocking temperatures (maximum values of $595^{\circ}-$ $620^{\circ} \mathrm{C}$ ). After tilt corrections, which vary among sites (Table 1 ), the site-mean directions are better grouped $\left(\kappa_{\mathrm{corr}}=30.6, \kappa_{\mathrm{in} \mathrm{situ}}=\right.$ 16.7) and define a north directed, moderate inclination population (Figure 5a). Site GW27 yields an anomalous south-southeast declination, subhorizontal positive inclination mean direction, and this result was excluded from the grou- mean calculations, as it lies more than two angular standard deviations away from the overall group mean.
[22] The tilt-corrected group mean from the 12 accepted sites (7 normal polarity, 5 reversed polarity) has a north declination, moderate inclination magnetization (Table 2; $D=359^{\circ}, I=+46^{\circ}$, $\alpha_{95}=8^{\circ}$ ). This mean is similar to and within statistical error of the mid-Miocene expected direction $\left(358^{\circ},+55^{\circ}, \alpha_{95}=6.0^{\circ}[\right.$ Mankinen et al., 1987]) and is consistent with no statistically significant vertical axis rotation (rotation $(R)=1.2^{\circ} \pm 14.1^{\circ}$ ) of the northern Greenwater Range since $7 \mathrm{Ma}$. The slightly shallower inclination (flattening $(F)=8.3^{\circ} \pm 10.1^{\circ}$ ) is also statistically insignificant and may result from inability to adequately remove a younger normal polarity overprint from the reversed polarity sites.

[23] As both normal and reversed polarity volcanic flow means were obtained from different levels within the volcanic sequence, the eruption of the Greenwater Volcanic rocks spanned at least two polarity reversals. A reversal test [McFadden and McElhinny, 1990] was conducted on the corrected remanence directions to test whether the set of 12 sites were drawn from the same dualpolarity population. The results of the test yield an indeterminate result. The critical angle between the two populations is $11.2^{\circ}$; the angle between the mean of normal set and the mean of the reversed set is $18.6^{\circ}$. The outcome of this test probably reflects the relatively small number of independent field readings and/or a bias imposed on the data set due to an unremoved magnetization. The former interpretation appears more plausible, as most reversed polarity site means, when inverted through the origin, 


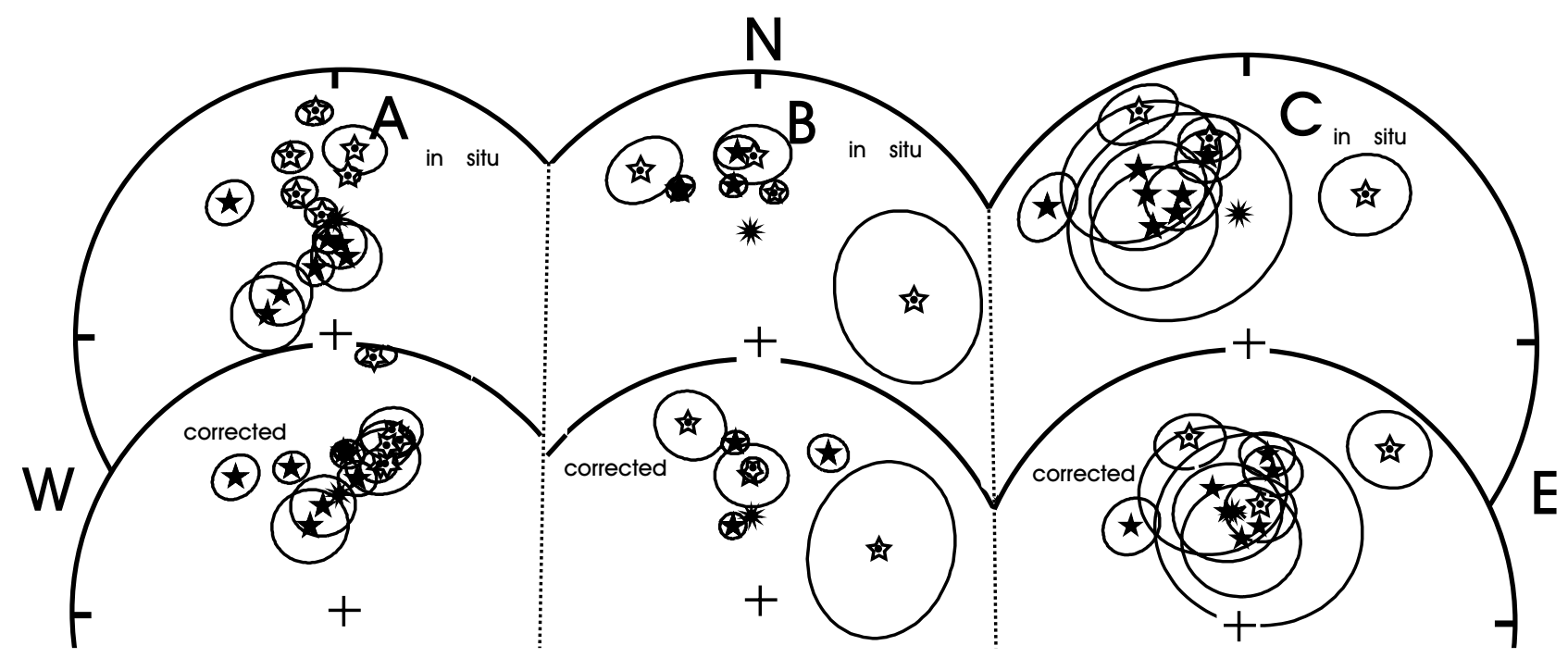

Figure 5. Partial equal-area projections of Greenwater Range site-mean directions (stars) with projected $\alpha_{95}$ confidence cones (ellipse). Solid symbols represent true lower hemisphere projections. Open symbols with dots are projections of site-mean directions on the lower hemisphere that have been inverted through the origin. Top (bottom) diagram shows in situ (corrected) site mean directions. (a) Greenwater Volcanics site mean directions, (b) Browns Peak pyroclastic rock site mean directions, and (c) Chocolate Sundae Mountain site-mean directions. Solid sun is Miocene expected direction (from Miocene paleomagnetic pole of Mankinen et al. [1987]). See Table 1 for site bedding corrections used.

have declinations that are more eastward than the normal polarity site means.

4.1.2. Browns Peak pyroclastics. [24] The 7 Ma Browns Peak pyroclastic rocks were collected via helicopter in a gently dipping section in the northeast part of the central Greenwater Range (Figure 2) [Thompson et al., 1993]. Samples were collected from seven sites in pyroclastic rocks and from one site in a mafic dike cutting the pyroclastic rocks. Sites were distributed to span as much of the section as possible, with four sites obtained near the base of the section, on the northwest side of the peak, and four sites just below the south plateau along the southern flank of the peak (Figure 2). A total of 85 specimens from the eight sites were demagnetized. Three site means were rejected from the groupmean calculation on the basis of uninterpretable demagnetization behavior (GW90), a mean direction $>45^{\circ}$ away from the overall group-mean direction (GW89), or because of unacceptably high dispersion (GW74) $\left(\alpha_{95}=23.9^{\circ}\right.$ and $\left.\kappa=15.7\right)$. Site GW74 is located near the top of Browns Peak, and the high dispersion at this site is most easily explained as a lightning-induced isothermal remanent magnetization (IRM), as the intensity of the NRM is 3 orders of magnitude greater than the other five sites in pyroclastic rocks. The four accepted site means in pyroclastic rocks were used to calculate an overall group-mean direction. One site yielded a positive, and three sites yielded a reverse polarity magnetizations (Figure 5c). The dual-polarity magnetizations from the Browns Peak pyroclastic section show that emplacement of these rocks spanned at least one polarity reversal. In addition, the mafic dike cutting the pyroclastic sequence also yielded normal polarity results with a similar direction; however, this site mean was not included in the overall group-mean calculation. The four accepted sites yield predominately single-component, well-grouped magnetizations with medium to high coercivities (median destructive inductions ranges of 20 to $50 \mathrm{mT}$; Figure $4 \mathrm{~b}$ ) and high maximum laboratory unblocking temperatures $\left(525^{\circ}-625^{\circ} \mathrm{C}\right)$. In situ site-mean directions, after reversed polarity data are inverted through the origin, are of northwest declination and moderate positive inclination (Figure 5c). After minor structural corrections between-site dispersion $\left(\kappa_{\text {corr }}=46.8, \kappa_{\text {in situ }}=26.9\right)$ decreases (Figure 5c) and the data yield a north-northwest declination and moderate positive inclination magnetization (Table $2 ; D=343^{\circ}, I=$ $\left.49^{\circ}, \alpha_{95}=11.3^{\circ}\right)$. The discordance of this group mean, in declination and inclination, to the Miocene expected direction, with rotation $(R)$ of $-14.3^{\circ}\left( \pm 19.3^{\circ}\right)$ and an inclination shallowing $(F)$ of $+5.3^{\circ}\left( \pm 12.8^{\circ}\right)$, is not statistically significant.

4.1.3. Greenwater Range intrusions. [25] A total of 35 sites were collected in synrift intrusive rocks in the central Greenwater Range (Figure 2). Fourteen sites were collected in the $9.76 \mathrm{Ma}( \pm 0.25 \mathrm{Ma})$ Chocolate Sundae Mountain granite, a megascopically undeformed monzonite granite in the west central Greenwater Range [Holm et al., 1992]. An additional 21 sites were collected in compositionally similar plutons exposed north of the Chocolate Sundae Mountain granite that crop out along the north and south sides of Deadman's Pass (Figure 2), hereinafter referred to informally as the Deadman's Pass pluton. These intrusions are assumed to be similar in age as the Chocolate Sundae Mountain granite.

[26] A total of 102 specimens were demagnetized from the 14 sites in the Chocolate Sundae Mountain granite. Four sites were excluded from the group-mean calculation on the basis of erratic demagnetization data (GW34) or because the sample magnetizations were too dispersed $(k$ values $<10)$ (GW11, GW40, and GW43). The 10 accepted site-mean directions include six sites in monzonite granite, three sites in dikes, and one site in an immediately overlying vitrophyre. These results give predominately single-component, well-grouped magnetizations with high coercivities (median destructive fields range between 20 and 75 $\mathrm{mT}$; Figure $4 \mathrm{c}$ ) and high maximum laboratory unblocking temperatures $\left(550^{\circ}-600^{\circ} \mathrm{C}\right)$. In situ site-mean directions are of both positive (five monzonite granite, two felsic dike) and reversed (one monzonite granite, one mafic dike, one vitrophyre) polarity magnetizations. The dual-polarity data suggest that the pluton cooled slowly over an interval that spanned at least one polarity reversal. The in situ results from 10 sites (with reversed polarity sites inverted through the origin) are generally of northwest declination and moderate to shallow positive inclination. One site (GW36) yielded a northeast declination and shallow positive inclination (Figure 5a) and was omitted from group-mean calculation. 


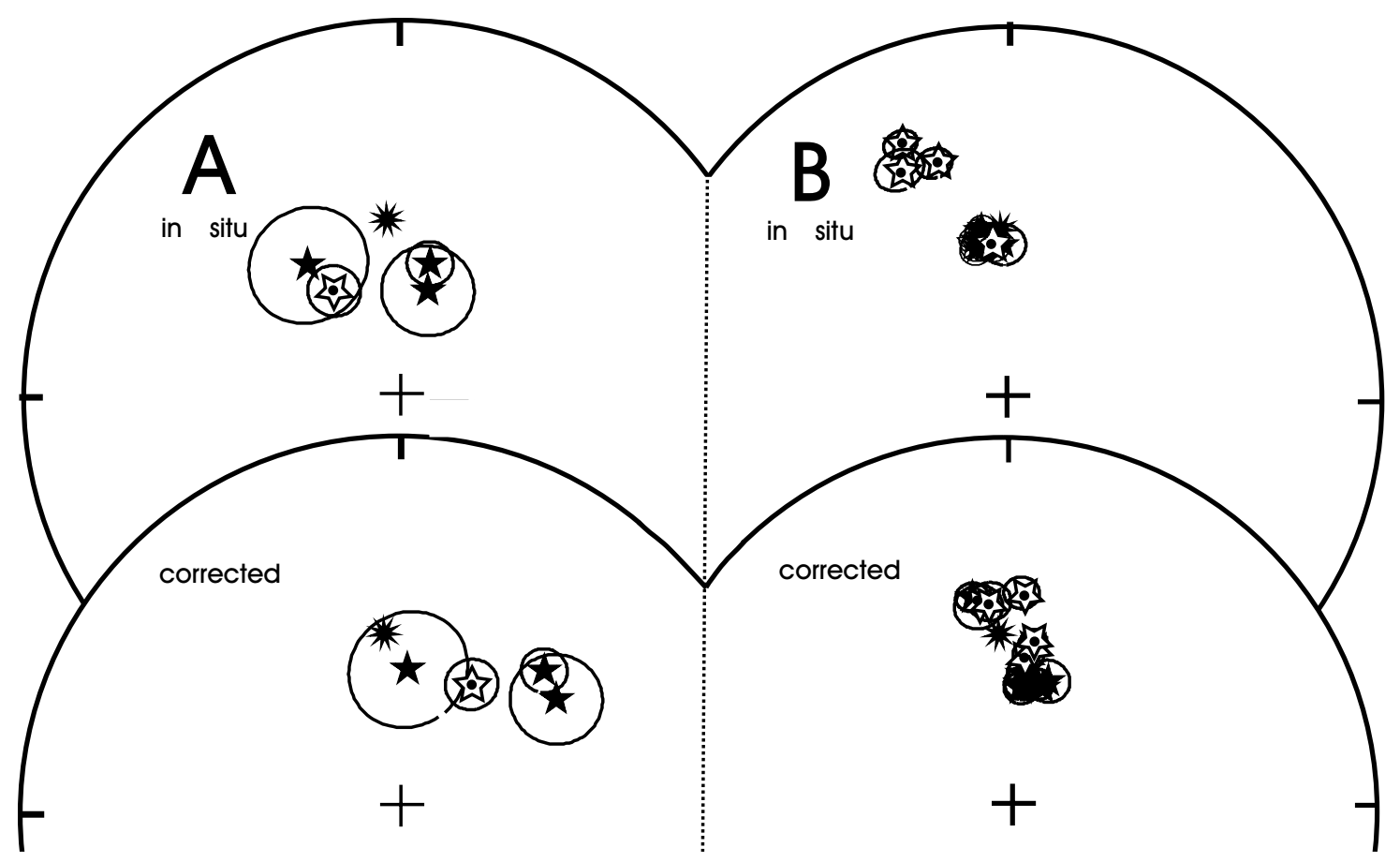

Figure 6. Partial equal-area projections of Black Mountain site-mean directions (stars) with projected $\alpha_{95}$ confidence cones (ellipse). Solid symbols represent true lower hemisphere projections. Open symbols with dots are projections of site-mean directions on the lower hemisphere that have been inverted through the origin. Top (bottom) diagram shows in situ (corrected) site-mean directions. (a) Black Mountain basalt site mean directions and (b) Funeral Peak basalt site mean directions. Asterisk is Miocene expected direction (from Miocene paleomagnetic pole of Mankinen et al. [1987]). See Table 1 for site bedding corrections used.

[27] The 8.5-7.0 Ma Shoshone basalt flows disconformably overlie the Chocolate Sundae Mountain granite and strike, generally, north-south $\left(010^{\circ} \mathrm{E}\right)$ and dip $\sim 30^{\circ}$ to the east. These overlying basalt flows provide at least a minimum tilt correction for the paleomagnetic data from the pluton. After this tilt correction the corrected group-mean result from the pluton has north declination and moderate positive inclination (Table $2 ; D=358^{\circ}, I=+49^{\circ}, \alpha_{95}$ $=9.6^{\circ}$ ). It should be noted that removing the tilt of the overlying basalt flows does not necessarily take into consideration any prenonconformity tilting of the pluton. The fact that the wellgrouped, corrected mean direction is similar to a mid-Miocene expected direction suggests that only modest tilting of the pluton took place prior to the eruption of the basalt flows. Excluding the results from the three sites in dikes and one site in basal vitrophyre from the group-mean calculation does not significantly change the direction or dispersion of the group-mean direction $\left(D=355.4^{\circ}, I\right.$ $=52.9^{\circ}, \kappa=20.4, \alpha_{95}=17.3^{\circ}$; compare with values in Table 2 ). Overall, the group-mean result from the granite and the younger intrusive and pyroclastic rocks is very close to the Miocene expected direction, which we interpret as consistent with a history of modest east-side-down tilting of this part of the southern Greenwater Range since about $10 \mathrm{Ma}$ (Figure 5a).

4.1.4. Deadman's Pass pluton. [28] For the Deadman's Pass pluton, 113 samples from 21 sites were collected. Only nine samples from two sites yielded interpretable demagnetization results. The rejected samples yielded nonlinear and, in most cases, erratic trajectories on vector plots such that a site-mean direction could not be calculated. The paleomagnetic data from these rocks, unfortunately, provide little insight into the tectonic history of the area and they will not be discussed further.

[29] In summary, the acceptable paleomagnetic results from the Greenwater Canyon volcanic rocks show no discernible vertical axis rotation since $\sim 7 \mathrm{Ma}\left(R=+1.2^{\circ} \pm 14.1^{\circ}\right)$ and results from the Chocolate Sundae Mountain intrusion rocks show no net rotation
$\left(R=+0.3^{\circ} \pm 17 \cdot 9^{\circ}\right)$. Post-10 Ma deformation in the Greenwater Range is interpreted to have occurred by local horizontal axis tilting associated with crustal extension.

\subsection{Black Mountains}

4.2.1. Black Mountain basalts. [30] A collection of 43 samples of the 8.5-7.5 Ma Black Mountain basalts was obtained from four $\left(25^{\circ}\right)$ east dipping flows along the eastern flank of the Black Mountains (Figure 2) [Fleck, 1970]. These basalts are equivalent in age to the Shoshone Volcanics. Overall, the Black Mountain basalts demagnetized exceptionally well (Figure 4d).

[31] Three site means are of normal polarity, and one site mean is of reversed polarity (Table 1 and Figure 6a). All four sites yield well-grouped, single-component magnetizations with high coercivities (median destructive induction fields from 20 to $30 \mathrm{mT}$; Figure 4d) and distributed maximum laboratory unblocking temperatures $\left(\sim 570^{\circ} \mathrm{C}\right)$. After inverting the single reverse polarity site through the origin and correcting for the modest east dip $\left(23^{\circ}\right)$ of the flows, site-mean directions are east-northeast (three sites) and north (one site) declination and moderate inclination (Figure 6a). The corrected group mean from the four sites yields a northeast declination, moderate positive inclination magnetization (Table $2 ; D=$ $039^{\circ}, I=62^{\circ}, \alpha_{95}=14.3^{\circ}$ ). This group mean is discordant from the Miocene expected direction with an inferred rotation of $+41^{\circ}$ $\left( \pm 32.6^{\circ}\right)$ and an inclination steepening of $-6.9^{\circ}\left( \pm 15.6^{\circ}\right)$. The importance of this result is limited, however, in that only four independent(?) readings of the field could be obtained. It is possible that the moderately discordant paleomagnetic directions likely reflect either high-amplitude paleosecular variation or a transitional field during a reversal.

4.2.2. Funeral Peak basalts. [32] Samples of 7-5 Ma Funeral Peak basalt flows were collected from a southeast dipping $\left(15^{\circ}-27^{\circ}\right)$ eruptive sequence along the eastern flank of 


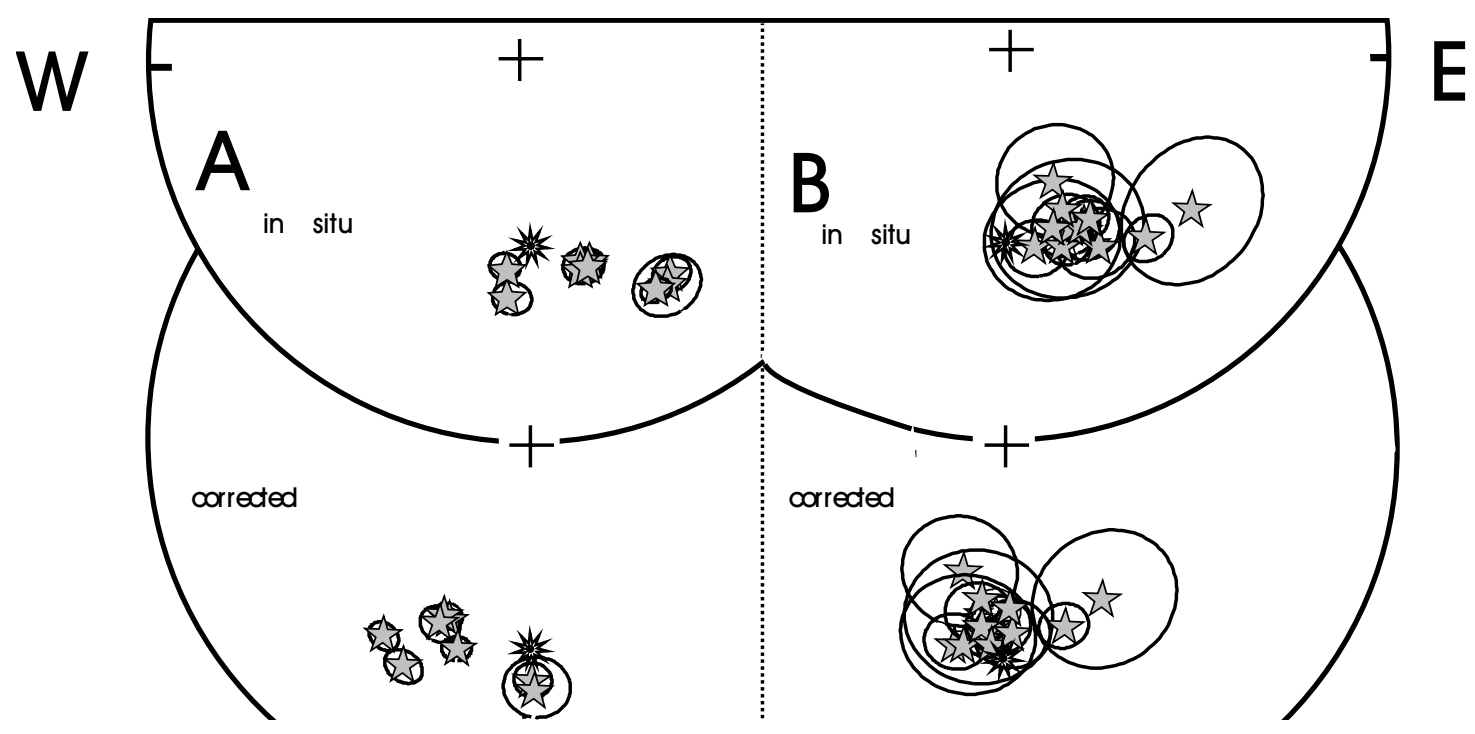

Figure 7. Partial equal-area projections of Panamint Mountains site-mean directions (stars) with projected $\alpha_{95}$ confidence cones (ellipse). Shaded stars represent true upper hemisphere projections. Top (bottom) diagram shows in situ (corrected) site-mean directions. (a) Dinosaur Ridge basalt site-mean directions and (b) Little Chief stock sitemean directions. Open asterisk with dot is Miocene expected direction (from Miocene paleomagnetic pole of Mankinen et al. [1987]). See Table 1 for site bedding corrections used.

the central Black Mountains (Figure 2) [Thompson et al., 1993]. A total of 15 sites were collected, with each site in a separate flow. All distinct flows of the sequence were sampled. A suite of 109 samples from the 15 sites was demagnetized. Overall, the Funeral Peak basalt flows, not surprisingly, demagnetized exceptionally well (Figure 4e).

[33] Acceptable site-mean directions are of both normal (nine sites) and reversed (six sites) polarity (Table 1 and Figure $6 \mathrm{~b}$ ). The 15 sites yield well-grouped, single-component magnetizations with high coercivities (median destructive fields from 30 to $50 \mathrm{mT}$; Figure $4 \mathrm{e}$ ) and distributed maximum laboratory unblocking temperatures $\left(560^{\circ}-580^{\circ} \mathrm{C}\right)$. After inverting reverse polarity data through the origin, in situ site-mean directions are of north to northwest declination and moderate positive inclination (Figure $6 \mathrm{~b}$ ). In order to test whether data from basalt flows represent samples drawn from the same dual-polarity population, the reversal test of McFadden and McElhinny [1990] was conducted on the corrected site means. The critical angle for the two populations is $7.5^{\circ}$; the angle between the mean of normal set and the mean of the reversed set is $7.5^{\circ}$. The results could be resolved to better than $10 \%$ but not better than $5 \%$ and indicate a positive reversal test at the $90 \%$ confidence level.

[34] Applying the appropriate correction for the modest southeast dip of the flows, site-mean directions are more north directed, with moderate positive inclinations (Figure 6b). We interpret the well-grouped characteristic magnetization of the 15 sites as a primary thermoremanent magnetization and that on the basis of the distribution of the polarities in the sequence, eruption of these flows spanned at least four polarity reversals. The corrected group-mean data from all 15 sites yield north declination, moderate steep inclination magnetizations (Table $2 ; D=$ $006^{\circ}, I=62^{\circ}, \alpha_{95}=4.0^{\circ}$ ). The results are exceptionally well defined and are not statistically discordant with the late Miocene expected direction $R=+8.3^{\circ}\left( \pm 11.4^{\circ}\right)$ and $F=-6.2^{\circ}\left( \pm 7.4^{\circ}\right)$. We interpret the results from the Funeral Peak basalts to indicate minimal clockwise vertical axis rotation of the eastern Black Mountains since $\sim 5 \mathrm{Ma}$.

[35] In summary, paleomagnetic data from the eastern Black Mountains are interpreted to indicate minimal post-5 Ma vertical axis rotation of the range and the field relations reveal only minor horizontal-axis tilting associated with northwest directed extension.

\subsection{Panamint Mountains}

4.3.1. Dinosaur Ridge basalts. [36] Samples from vesicular basalts were collected from nine sites in each of the total nine basalt flows that crop out near "Dinosaur Ridge" (informal name of Hunt and Mabey [1966]) along the eastern base of the Panamint Mountains (Figure 3). The precise age of the basalt is somewhat uncertain. A whole rock ${ }^{40} \mathrm{Ar} /{ }^{39} \mathrm{Ar}$ analysis (B. Wernicke and K. Hodges, unpublished data, 1994) yielded a complex spectrum, where most of the gas was released in two increments, with dates of $10.2 \pm 0.2 \mathrm{Ma}$ and $10.5 \pm 0.3 \mathrm{Ma}$. However, a biotite K-Ar date on a silicic tuff stratigraphically beneath the basalts is $9.0 \pm 0.1 \mathrm{Ma}$ (W. Hildreth, unpublished data, 1983). The source of the discrepancy has not been identified, and therefore, although it is probable that the basalts are $\sim 9-11 \mathrm{Ma}$, the possibility remains that they are younger than $\sim 9$ Ma. Sixty-five specimens from the nine sites provided interpretable demagnetization results. The nine sites yield very well grouped, single-component magnetizations with high coercivities (median destructive fields of 10-40 mT; Figure 4f) and distributed maximum laboratory unblocking temperatures (to between $560^{\circ}$ and $580^{\circ} \mathrm{C}$ ). After correcting for the flow layering $\left(196^{\circ} / 39^{\circ}\right.$, east dip), the corrected group-mean paleomagnetic data from the nine sites (Table 1 and Figure 7a) yield south to southwest declination, moderate negative inclination magnetizations (Table 2; $\left.D=205^{\circ}, I=-44^{\circ}, \alpha_{95}=9^{\circ}\right)$. The overall mean is somewhat discordant to the expected reversed polarity late Miocene direction, with an inferred rotation of $+27.6^{\circ}\left( \pm 15.2^{\circ}\right)$ and a flattening of $-10.7^{\circ}\left( \pm 11.2^{\circ}\right)$. At face value, these data can be interpreted to suggest modest clockwise rotation of the basalt section. The VGP angular dispersion of the nine site means is $15.8^{\circ}$, in comparison to values between $13^{\circ}$ and $15^{\circ}$ predicted by paleosecular variation model $\mathrm{G}$ for this latitude [McFadden et al., 1991]. Although the VGP angular dispersion of the site means is similar to predicted values, together the smaller angular dispersion of the data set and uniform reverse polarity, however, may indicate that the group-mean 


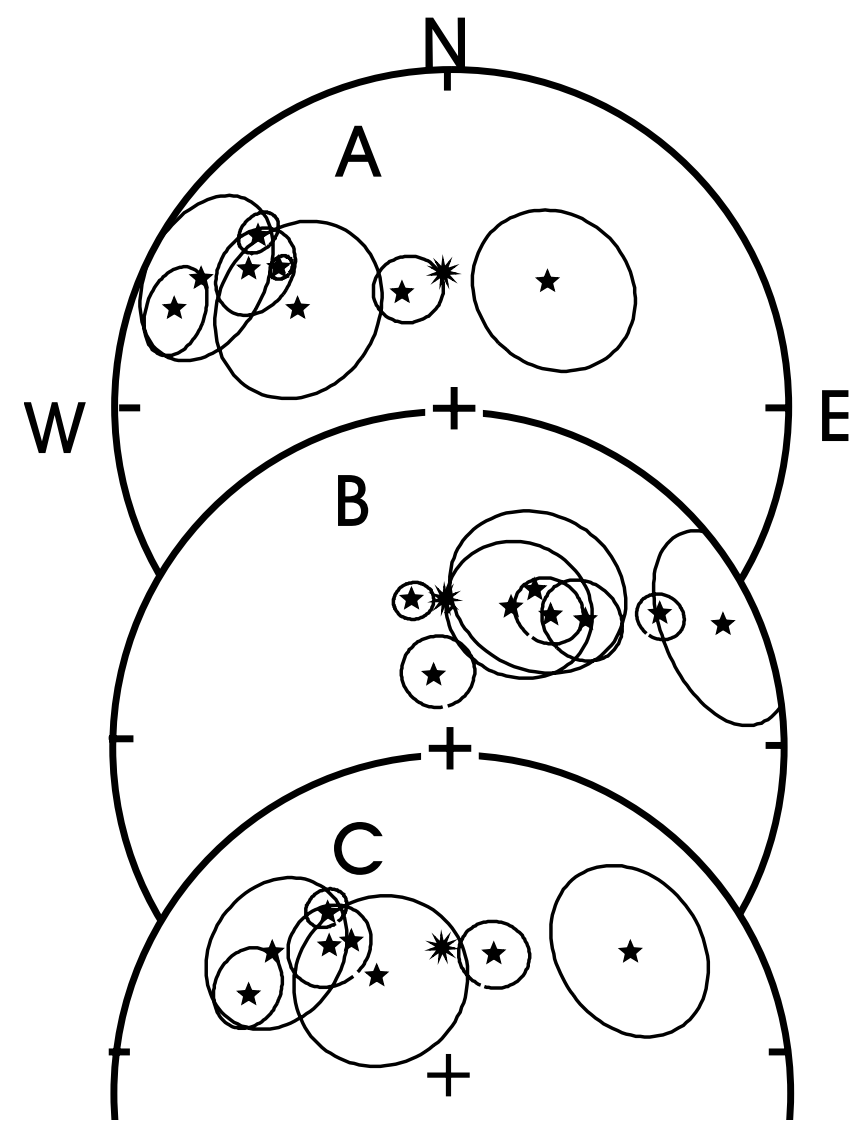

Figure 8. Partial equal-area projection of site mean directions (stars) with projected $\alpha_{95}$ confidence cones (ellipse) from Ordovician Pogonip strata in the Panamint Mountains. Solid stars represent lower hemisphere projections. Asterisk is Miocene expected direction. (a) In situ site mean directions. At least two structural corrections are plausible. (b) Bedding corrections based on bedding orientations at individual sampling site and (c) a regional correction based on the orientation of the Tertiary lava flows on the east flank of the range. Solid sun is Miocene expected direction (from Miocene paleomagnetic pole of Mankinen et al. [1987]). See Table 1 for site bedding corrections used.

result does not represent a time-averaged field direction and that the estimates of statistically significant rotation is not highly robust.

4.3.2. Little Chief stock. [37] Samples of the $10.6 \mathrm{Ma}$ Little Chief stock were collected from nine sites spanning the northern and southern phases of the intrusion, located east of Telescope Peak (Figure 3) [Albee et al., 1981; McKenna, 1986]. Additional samples were collected from a genetically related granitic dike (one site) and contact metamorphosed carbonate host rocks (two sites). A collection of 118 samples was obtained from the 12 sites and demagnetized. Three sites were rejected as samples did not provide interpretable demagnetization results (site LC21) or their dispersion at the site level was unacceptably high (sites LC18, ZN1). The nine accepted site-mean directions are all of reversed polarity (Table 1 and Figure $7 \mathrm{~b}$ ) and yield well-grouped magnetizations with high coercivities (median destructive fields from 35 to $60 \mathrm{mT}$ for specimens with essentially single-component magnetization; Figure $4 \mathrm{~g}$ ) and high maximum laboratory unblocking temperatures $\left(550^{\circ}-\right.$ $\left.570^{\circ} \mathrm{C}\right)$. All in situ site-mean directions are of south-southeast declination and moderate negative inclination (Figure 7b).

[38] The tilt correction for the Little Chief stock paleomagnetic data is best estimated by the tilt of gently east tilted Neoproterozoic sedimentary wall rocks to the intrusion. As described in section 4.3.1, middle and upper Miocene (10.5 to $\sim 9 \mathrm{Ma})$ volcanic strata along the eastern flank of the Panamints (including the Dinosaur Ridge basalts) generally rest disconformably or with gentle angular unconformity on pre-Tertiary strata [Hunt and Mabey, 1966; McKenna and Hodges, 1990; Wernicke, 1993]. Where discordant, pre-Tertiary strata are steep $\left(60^{\circ}\right)$ and dip more steeply to the east (by up to $25^{\circ}$ ) than the Tertiary.

[39] These relations suggest that at large scale the miogeoclinal wall rocks of the stock were subhorizontal prior to deposition of Miocene volcanic rocks during intrusion of the Little Chief stock. On the basis of a mean strike of $358^{\circ}$ and mean dip of $22^{\circ} \mathrm{E}$ for the surrounding Johnnie Formation strata [Albee et al., 1981] the nine site-mean directions provide an overall corrected group mean of south declination and moderate negative inclination (Figure $7 \mathrm{~b}$ and Table 2; $D=181^{\circ}, I=-51.7^{\circ}, \alpha_{95}=6^{\circ}$ ). This group mean is nearly identical to the Miocene reversed polarity expected direction, with a statistically insignificant rotation of $+3.4^{\circ}\left( \pm 13.1^{\circ}\right)$ and a flattening of $+3.3^{\circ}\left( \pm 8.9^{\circ}\right)$. Although up to $25^{\circ}$ of tilt may have occurred prior to $10.5 \mathrm{Ma}$ along the east flank of the antiform, this amount of tilt probably did not affect the core, where presumably tilt developed in similar proportion, not magnitude, as the antiform developed. If as much as a third of the tilt occurred prior to intrusion of the stock (corresponding to $20^{\circ}$ of $60^{\circ}$ tilt along the flank being prevolcanic), then the tilt correction would be as large as $29^{\circ}$. This value, which we consider a maximum, also yields groupmean direction close to the Miocene direction, $D=194^{\circ}, I=$ $-50^{\circ}$, with $R=+16^{\circ}$ and $F=+5^{\circ}$. The late Miocene deformational history of the central Panamint Mountains is consistent 
with modest east tilting of the range associated with northwestward translation of the range since $\sim 10$. 6 Ma [Niemi et al., 2001].

4.3.3. Paleozoic carbonate strata. [40] Samples from carbonate beds of the Ordovician Pogonip Group were collected at eight sites, having somewhat diverse, yet uniformly east dipping bedding orientations, along the eastern limb of the Panamint Mountains antiform near Tucki Mountain. The eight sites were chosen to cover a large north-south span within the range and to provide a range of bedding orientations to attempt to evaluate the antiquity of the magnetization using a fold test. A total of 65 samples were demagnetized from the eight sites. Progressive thermal demagnetization beyond $\sim 140^{\circ}-200^{\circ} \mathrm{C}$ generally yielded a linear trajectory toward the origin on vector projections, allowing the isolation of a stable endpoint magnetization for most samples (Figure 4h). The stable endpoint magnetizations had maximum unblocking temperatures between $\sim 300^{\circ} \mathrm{C}$ and $400^{\circ} \mathrm{C}$ (Figure $4 \mathrm{~h}$ ), suggesting that pyrrhotite is an important remanence carrier. In AF demagnetizations, most samples reveal median destructive fields $>50 \mathrm{mT}$, suggesting that a high-coercivity cubic phase carries the remanence.

[41] With the exception of one site (OP8), the Ordovician Pogonip Group carbonate rocks yield in situ mean directions that are of west-northwest to northwest declination and moderate positive inclination (Table 1 and Figure 8). Two of these eight sites have relatively high dispersion yet yield site means that are similar to those from sites with lower dispersions. Site OP8 yields a mean direction of northeast declination and moderate positive inclination. The overall group mean for six of the eight sites (excluding OP3 and OP8) yields an in situ result of $D=303^{\circ}, I$ $=+29^{\circ}, \alpha_{95}=11^{\circ}$ (Figure 8a).

[42] We applied two different tilt corrections to the data from the Pogonip strata to attempt to evaluate the age of the characteristic magnetization relative to deformation and thus to determine if this magnetization could be used to assess structural deformation. The basic rationale for applying a correction is the observation that most of the in situ data do not resemble an expected late Tertiary field direction. One tilt correction is based on bedding orientations at each site in Pogonip Group strata, which vary by more than $50^{\circ}$ $\left(37^{\circ}-108^{\circ}\right.$, east-down tilt) (see Table 1$)$. The second tilt correction assumes that the magnetization is not primary, yet was acquired prior to Neogene extension, and uses the orientation of the Tertiary basalt section at Dinosaur Ridge $\left(016^{\circ} / 39^{\circ}\right.$, east dip). Although group-mean results vary depending on the structural correction applied (Figures $8 \mathrm{~b}$ and $8 \mathrm{c}$ ), both corrections result in an overall distribution of site means that is more north directed and of steeper positive inclination. Application of the corrections based on bedding attitudes at each site results in a slight increase in overall dispersion $\left(\kappa_{\text {insitu }}=15.7 ; \kappa_{\text {corr }}=11.8\right)$ and yields a group mean of northeast declination and moderate positive inclination (Table 2; $D$ $=028^{\circ}, I=45^{\circ}, \alpha_{95}=11^{\circ}$ ). A uniform, regional tilt correction, using the orientation of the Tertiary basalt section at Dinosaur Ridge, yields a regional corrected group-mean direction of $D=$ $322^{\circ}, I=+65^{\circ}, \alpha_{95}=11^{\circ}$ (Figure 8c) and, of course, does not change the dispersion. Regardless of the applied correction, it is clear that the magnetization in the Pogonip strata is not primary because inclinations (relative to bedding) are considerably steeper than those expected for the Ordovician on the basis of the early Paleozoic part of the North American apparent polar wander path [e.g., Van der Voo, 1993].

[43] In summary, paleomagnetic data from the Little Chief stock in the Panamint Mountains are interpreted to indicate little statistically significant post-10.6 Ma range-scale vertical axis rotation of the central part of the range, assuming a modest east tilt of the range since pluton emplacement. The Panamint Mountains appear to have been transported en mass laterally to their current location and have experienced only modest range-scale horizontal axis tilting associated with crustal extension, with possible (but not statistically demonstrable) modest clockwise rotation of the northern part of the range.

4.3.4. Summary of paleomagnetic data. [44] Our data indicate that both the Greenwater Range and eastern Black Mountains have not rotated significantly since circa $10 \mathrm{Ma}$ and 5 $\mathrm{Ma}$, respectively, and westward translation of the Panamint block was accompanied by modest east-side-down tilting in the absence of significant vertical axis rotation of the high, central part of the range since about 10.6 Ma.

\section{Discussion}

[45] The mid-Miocene and younger tectonic evolution of the central Death Valley extended terrane has been evaluated on the basis of structural and stratigraphic relations [Snow and Wernicke, 1989; Snow and Prave, 1994], geochronology [Wright et al., 1991; Holm et al., 1994a; Thompson et al., 1993; Holm et al., 1992; McKenna and Hodges, 1990; McKenna, 1986; Haefner, 1974; Fleck, 1970], and paleomagnetic data [this study; Petronis et al., 1997; Holm et al., 1993; Snow et al., 1993]. Field observations demonstrate that both extensional and strike-slip fault systems have influenced the late Miocene and younger development of the region since rapid extension began at about $14 \mathrm{Ma}$. The paleomagnetic data from this study place limits on the expected magnitudes of vertical axis rotation of crustal blocks, as recently proposed in a model that invokes significant rotation (up to $30^{\circ}$ clockwise) of ranges across the central Death Valley region [Serpa and Pavlis, 1996].

[46] According to this model, tectonism occurred in two phases, one from 14 to $6 \mathrm{Ma}$ and the younger from $6 \mathrm{Ma}$ to the present. The later phase is characterized by development of the Death Valley, Panamint Valley, and Owens Valley fault systems and by mafic volcanism. For the young phase of deformation the Cottonwood Mountains are restored to a position over the northwest Panamint Mountains, to close the Nova basin, and the Panamint Mountains are restored directly adjacent to the Black Mountains to close the central Death Valley basin. The closure of the basins is accompanied by $15^{\circ}$ of counterclockwise vertical axis rotation of the Panamint Mountains [Serpa and Pavlis, 1996, Figure 2b]. To the east, the Black Mountains and Greenwater Range are tilted eastward, to varying degrees, without range-scale vertical axis rotation.

[47] For the earlier period the Panamint Mountains (the hanging wall of the Amargosa Fault system) are restored above the Black Mountains crystalline core, placing the latter to midcrustal depths [Holm et al., 1992; Asmerom et al., 1990]. In addition to eastward restoration the Panamints are back rotated an additional $10^{\circ}-20^{\circ}$ counterclockwise (depending on the reconstruction chosen) so as to accommodate presumed thrust correlations, in particular the correlation of the Panamint thrust at Tucki Mountain with the Clery thrust in the southeastern Funeral Mountains and the Butte Valley thrust in the southern Panamints with the Chicago Pass thrust in the northern Nopah Range [Serpa and Pavlis, 1996, Figures 2c and 2d].

[48] The transtensional shear hypothesis conflicts with several important structural and stratigraphic tests. These include (1) the proximity $(<20 \mathrm{~km})$ of the southern Cottonwood Mountains and the northern Nopah Range at $11 \mathrm{Ma}$, as indicated by middle Miocene, Cottonwood-derived proximal fan deposits in the Chicago Pass area [Niemi et al., 2001]; (2) the correlation of the Panamint thrust with the Chicago Pass, not the Clery thrust, as suggested by others [Wernicke et al., 1988, ; Wernicke, 1993; Snow and Wernicke, 2000]; (3) the covariance of regional paleocurrent directions in Lower Cambrian strata with Mesozoic structural trends, indicating no differential rotation of the Panamints, Nopah, and Resting Spring Ranges [Snow and Prave, 1994]; and (4) paleomagnetic data from the southern Cottonwood, Funeral, and 


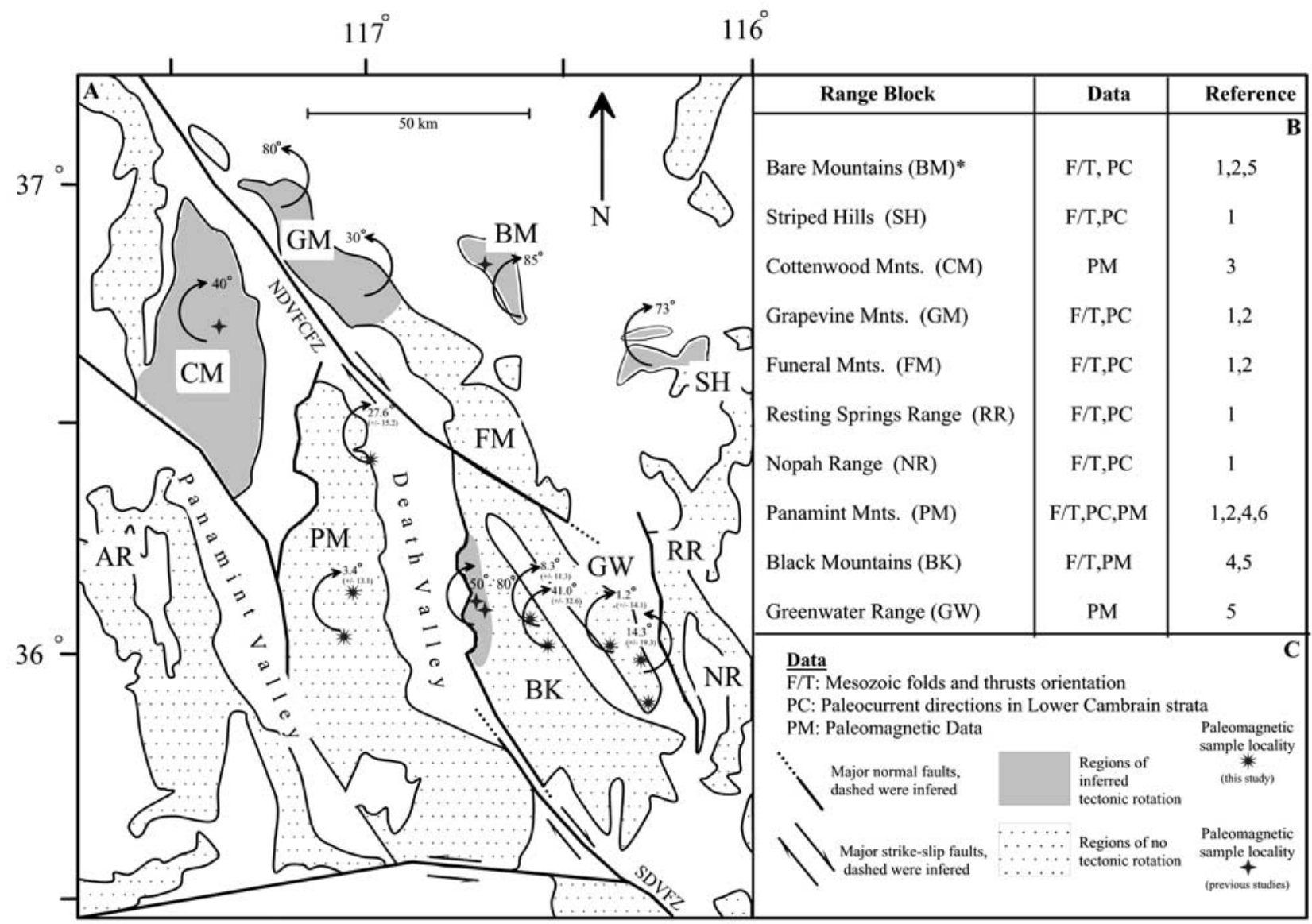

Figure 9. Simplified map of the Death Valley region showing areas of inferred tectonic rotation (shaded areas) and little to no tectonic rotation (stippled areas). Basis (a) Orientation of major Mesozoic folds and thrusts; (b) Paleocurrent directions in Lower Cambrian strata; and (c) Paleomagnetic data. References: 1, Snow and Prave [1994]; 2, Snow and Wernicke [1989]; 3, Snow et al. [1993]; 4, Holm et al. [1993]; 5, Stamatakos et al., 1998; 6, this study. NDVFCFZ, northern Death Valley-Furnace Creek fault zone; SDVFZ, southern Death Valley fault zone; GFZ, Garlock fault zone. Asterisks, data from Bare Mountain are conflicting. Map modified from Snow and Prave [1994].

Grapevine Mountains [Snow et al., 1993; J. K. Snow and J. W. Geissman, unpublished data, 1995] that support Snow and Prave's [1994] conclusions (Figure 9). In addition to these difficulties the paleomagnetic data reported here from the Greenwater Range and Black Mountains, and to a lesser extent the data from the Panamint Mountains, are not consistent with significant vertical axis rotation, of either sense, of these ranges.

\subsection{Panamint Mountains}

[49] The kinematics of translating the Panamint Mountain structural block to its current location are integral to any reconstruction of the central Death Valley region. One possible hypothesis, considering a transtensional model [Serpa and Pavlis, 1996], is that during the early phase of deformation the Panamint Mountains were translated northwestward to a position adjacent to the present-day western margin of the Black Mountains. During this early phase of translation the Panamint structural block is proposed to have rotated some $10^{\circ}-20^{\circ}$ in a clockwise fashion. Assuming that the magnetizations are primary, paleomagnetic results from the 10.6 Ma Little Chief Stock and $11 \mathrm{Ma}($ ?) Dinosaur Ridge basalts should reflect at least a part of the rotational component and be discordant in declination with respect to a mid-Miocene expected direction. Characteristic magnetizations in the Ordovician Pogonip Group strata are secondary and may have been acquired during Cretaceous contractile deformation. In principle, the magnetizations should record the full magnitude of rotation, as well as potentially earlier phases of deformation that affected the region since acquisition, although we recognize that the interpretation of these data is ambiguous.

[50] The data from the Little Chief stock provide no statistically significant evidence of vertical axis rotation. The overall group mean is restored to a value similar to a reversed polarity midMiocene expected direction by simply restoring the east dip of Neoproterozoic miogeoclinal strata to horizontal. The in situ result from the Little Chief stock, of south-southeast declination and moderate negative inclination, is notably consistent with modest counterclockwise, rather than clockwise rotation, and so any argument for a substantial clockwise rotation (e.g., $>20^{\circ}$ ) would require a tilt correction that is based on moderate to steeply east dipping strata. The group-mean result from the Dinosaur Ridge basalt section is consistent with a modest amount of clockwise rotation, but the reliability of this result is questionable, as all nine lava flows available for sampling are of reverse polarity and basically yield minimally two and at most four clusters of flow means. In general, one population ( $n=3$ sites) is similar to the reversed polarity expected direction while the other population $(n=$ 6 sites) is discordant in a clockwise sense to the expected direction. On the basis of the mean discrimination test of McFadden and Lowes [1981] the two populations are statistically distinct at $>99 \%$ probability.

[51] The data from the Pogonip Group strata are less straightforward to interpret. The in situ site means from seven of the 
eight sites, of northwest to west-northwest declination and moderate shallow positive inclination, are most readily explained by a moderate, east-side-down tilting of a normal polarity magnetization acquired during and/or after folding of these strata, which took place some time between the Permian and the Late Cretaceous [Snow and Wernicke, 1989, and references therein]. Our preferred interpretation of the available data is that the Ordovician strata were remagnetized in mid to late Mesozoic time and that the overall distribution of the data reflects eastside-down tilting of these rocks after magnetization acquisition. The exact process of remagnetization is not clear; it is possible that the secondary magnetization is synfolding in age, which might explain the high dispersion of the data. Given the uncertainty in the exact age of magnetization in these rocks and the possible range of postmagnetization acquisition structural corrections that could be applied, we do not emphasize these results in our overall interpretation of the late Cenozoic evolution of the Death Valley area. Although we cannot refute the possibility that vertical axis rotation of the Panamint Mountains occurred before emplacement of the Little Chief stock, paleomagnetic data from younger rocks, in particular the Little Chief stock, suggest that the Panamint Mountains did not experience appreciable vertical axis rotation from about 10.6 Ma to the present. We recognize that at face value, the limited data from the Dinosaur Ridge basalts yield a rotation estimate of $+27.6^{\circ}\left( \pm 15.2^{\circ}\right)$ and a flattening of $-10.7^{\circ}\left( \pm 11.2^{\circ}\right)$. The range thus appears to have been translated westward from a position atop the Black Mountains to its current location with only modest east-down tilting.

\subsection{Black Mountains}

[52] Paleomagnetic data from the Black Mountains include results from a 11.7 Ma mafic batholith, 9 to 6.5 Ma plutons and mafic and felsic dikes [Holm et al., 1993], as well as younger basalt flows (this study). Holm et al. [1993] proposed a kinematic sequence based on the paleomagnetic data from the Black Mountains that involved (1) southeast-side-down tilting $\left(20^{\circ}-70^{\circ}\right)$ of the range and folding of the turtlebacks between 11.6 and $8.7 \mathrm{Ma}$, (2) exhumation through a westward migrating rolling hinge between 8.7 and $6.5 \mathrm{Ma}$, and (3) a $50^{\circ}-80^{\circ}$ vertical axis clockwise rotation of the turtleback faults due to oroclinal folding between 6.5 Ma and the present. The undeformed silicic plutons and mafic to silicic dikes ranging in age from $\sim 9$ to $6.5 \mathrm{Ma}$ [Holm et al., 1992; Holm, 1995] that intrude the diorite yield inclinations similar to expected Miocene direction. Declinations, however, are statistically discordant and suggest that large-scale $\left(50^{\circ}-80^{\circ}\right)$ clockwise rotation of the crystalline core occurred late in the deformational history of the Black Mountains, presumably in response to postunroofing dextral shear along the Death Valley fault zone [Holm et al., 1993].

[53] The eastern margin of the Black Mountains is overlain by the 8.5-7.5 Ma Black Mountain and the 5-4 Ma Funeral Peak basalts, which dip gently to the east [Wright et al., 1991]. The four available flows in the 8.5-7.5 Ma Black Mountains basalts yield a group mean that is discordant to the expected direction in a clockwise sense; however, as discussed above, these results most likely reflect an unresolved field bias as only four independent directions could be obtained. The Funeral Peak basalts provide a relatively robust, dual-polarity data set and yield a corrected group mean similar to the expected late Miocene direction.

[54] In sum, the data from the 5-4 Ma volcanic rocks show no evidence of substantial rotation of the eastern part of the Black Mountains since $5 \mathrm{Ma}$, and results from the Black Mountains basalts are, unfortunately, inconclusive. The question then becomes, does the large apparent post-6.5 Ma clockwise rotation of the crystalline core also affect the eastern part of the range? If so, it would require rotation rates of some $50^{\circ}-80^{\circ} / \mathrm{Myr}$ during this time. We suggest that it is more probable that rotation is restricted to the crystalline core rocks in the western part of the range and that it has occurred over a much longer time period (from 6.5 Ma to present) in response to right shear on the Death Valley fault zone. Clockwise rotation of the Black Mountain crystalline core as suggested from the $8.7 \mathrm{Ma}$ Smith Mountain granite and younger intrusions [Holm et al., 1993], is interpreted to have resulted from right-lateral oroflexural bending of the northern Black Mountains along the southern Death Valley fault zone analogous to that seen in the Las Vegas Range adjacent to the Las Vegas Valley shear zone [Gillett and Van Alstine, 1982]. Localization of oroclinal bending to the westernmost part of the range may be reflected in the orientation of the range crest, where an east-west trending ridge is deflected in a right-shear sense into the northwest orientation of the Copper Canyon turtleback.

\subsection{Greenwater Range}

[55] Paleomagnetic data that bear on the extensional history of the Greenwater Range are provided by the $9.8 \mathrm{Ma}$ Chocolate Sundae Mountain Granite and the 7-5 Ma Greenwater volcanic rocks. The paleomagnetic data from the 7-5 Ma Greenwater volcanic rocks are consistent with modest east tilting, as restoring the flow layering of the volcanic rocks returns the group-mean direction to a value that is statistically indistinguishable from the expected Miocene direction. Moreover, the paleomagnetic results from the Chocolate Sundae Mountain granite are restored to the expected direction when the modest east tilt of the overlying volcanic rocks is removed. Overall, this suggests that the Greenwater intrusive and volcanic rocks shared none of the rotation recorded by the intrusive rocks in the Black Mountains and that the uplift and exhumation of the Chocolate Sundae Mountain granite did not involve significant deformation, prior to volcanism, and supports the hypothesis that the pluton was emplaced at very shallow crustal levels [Holm et al., 1993]. One possible explanation is that Greenwater Range intrusive and volcanic rocks were decoupled from intrusions in the Black Mountains, suggesting the presence of a separate crustal detachment layer between the Greenwater Range and the Black Mountains; there is no field evidence of structures capable of facilitating rotation of the Black Mountains independently of the Greenwater Range (Figure 9).

\section{Conclusions}

[56] The mid-Miocene Little Chief stock and Pogonip Group carbonate strata preserve a paleomagnetic record consistent with no appreciable vertical axis rotation of the Panamint Mountains. The nine flows comprising the upper(?) Miocene Dinosaur Ridge basalts, although suggestive of some rotation, are interpreted to represent an incomplete sampling of the field and thus yield inconclusive data, although the tilt-corrected mean is not consistent with moderate (e.g., $35^{\circ}$ ) clockwise rotation since emplacement. The lower Pliocene Funeral Peak basalts yield robust, dual-polarity paleomagnetic results that indicate no post-5 Ma vertical axis rotation of the eastern flank of the Black Mountains. As with the Dinosaur Ridge basalts, results from the Black Mountain basalts are inconclusive, most likely because of incomplete sampling of the field. The mid to late Miocene intrusive and upper Miocene to lower Pliocene volcanic rocks provide paleomagnetic results consistent with modest east tilting of the Greenwater Range and no evidence of vertical axis rotation. The results from the Browns Peak pyroclastic rocks are, unfortunately, highly dispersed yet suggest a component of counterclockwise, but statistically insignificant, rotation and overall may simply reflect an inadequate sampling of the geomagnetic field. Our results do not support the hypothesis that transtensional rotation of crustal-scale blocks occurred in the central Death Valley since 14 Ma [Serpa and Pavlis, 1996].

[57] Our current state of knowledge (depicted in Figure 9) on the regional pattern of vertical axis rotations of range-scale blocks within the Death Valley extended terrane is based on the orientation of Mesozoic structural markers, paleocurrent directions, and pale- 
omagnetic data. In the southern region, only localized clockwise rotation of the Black Mountains crystalline core is documented. This contrasts with significant and variable (both clockwise and counterclockwise) range-scale rotations in the northern part of the Death Valley region [Snow and Prave, 1994]. Inferred large magnitude, range-scale rotations in the northern Death Valley region were questioned by Stamatakos et al. [1998], who reported dual-polarity paleomagnetic data from Ordovician Pogonip Group strata at Bare Mountain. The overall demagnetization behavior of the Pogonip strata sampled at Bare Mountain is not dissimilar to that of the Panamint rocks examined in this study. Stamatakos et al. [1998] interpret magnetizations for seven sites in folded strata above the Meiklejohn thrust to be secondary and of Permian or Triassic age. Snow and Wernicke [2000] reinterpreted those data as Miocene in age and argue, on the basis of a different tilt correction for the in situ data, that the results could not be interpreted to suggest minimal rotation of Bare Mountain since the early Mesozoic. Given the evidence for such heterogeneous rotations in the Death Valley area, caution must be used before developing tectonic models based on assumptions about vertical axis rotations associated with extensional deformation.

[58] Acknowledgments. We thank Ren Thompson for use of his unpublished map of the central Death Valley extended terrane, John Miller and Dave Schneider for assistance sampling in sometimes inhospitable conditions, Zeke Snow for useful comments on a very early version of the manuscript, and the National Park Service for (limited) permission to work in Death Valley, California. Funded in part by a NSF grant (Wernicke and Geissman) and an American Chemical Society Grant (Holm). We greatly appreciate the efforts of the reviewers, Mark Hudson and Jon Hagstrum, which greatly clarified and improved an earlier version of the manuscript.

\section{References}

Albee, A. L., T. C. Labotka, M. A. Lanphere, and S. D. McDowell, Geologic map of the Telescope Peak Quadrangle, California, U.S. Geol. Surv. Geol. Quad. Map, GQ-1532, scale 1:62,500, 1981.

Asmerom, Y., J. K. Snow, D. K. Holm, S. B. Jacobsen, B. P. Wernicke, and D. R. Lux, Rapid uplift and crustal growth in extensional environments: An isotopic study from the Death Valley region, California, Geology, 18, 223-226, 1990.

Bennett, R. A., J. L. Davis, and B. P. Wernicke, Present-day pattern of Cordilleran deformation in the western United States, Geology, 27, 371-374, 1999.

Burchfiel, B. C., Structural geology of the Specter Range quadrangle, Nevada, and its regional significance, Geol. Soc. Am. Bull., 76, 175-192, 1965.

Burchfiel, B. C., and J. H. Stewart, "Pull-apart" origin of the central segment of Death Valley, California, Geol. Soc. Am. Bull., 77, 439-442, 1966.

Cemen, I., L. A. Wright, R. E. Drake, F. C. Johnson, Cenozoic sedimentation and sequence of deformational events at the southeastern end of the Furnace Creek strike-slip fault zone, Death Valley region, California, in Strike-Slip Deformation, Basin Formation, and Sedimentation, edited by K. T. Biddle and N. Christie-Blick, Spec. Publ. Soc. Econ. Paleontol. Mineral., 37, 127-141, 1985.

Fisher, R. A., Dispersion on a sphere, Proc. R. Soc.London, Ser. A, 217, 295-305, 1953.

Fleck, R. J., Age and tectonic significance of volcanic rocks, Death Valley area, California, Geol. Soc. Am. Bull., 81, 2807-2816, 1970.

Gillett, S. L., and D. R. Van Alstine, Remagnetization and tectonic rotation of upper Precambrian and lower Paleozoic strata from the Desert Range, southern Nevada, J. Geophys. Res., 87, 10,929-10,953, 1982.

Guth, P. L., Tertiary extension north of Las Vegas Valley shear zone, Sheep and Desert Ranges, Clark County, Nevada, Geol. Soc. Am. Bull., 92, 763-771, 1981.

Haefner, R., Geology of the Shoshone Volcanics, Death Valley region, eastern California, in Death Valley Region, California and Nevada, Field Trip Guideb., pp. 59-64, Cordilleran Sect., Geol. Soc. of Am., Boulder, Colo., 1974.

Hagstrum, J. T., and P. B. Gans, Paleomagnetism of the Oligocene Kalamazoo tuff: Implications for Middle Tertiary extension in east central Nevada, J. Geophys. Res., 94, 1827-1847, 1989.

Hamilton, W. B., Detachment faulting in the Death Valley region, California and Nevada, U.S. Geol. Surv. Bull., 1790, 51-85, 1988.
Hodges, K. P., J. D. Walker, and B. P. Wernicke, Footwall structural evolution of the Tucki Mountain detachment system, Death Valley region, southeastern California, in Continental Extension Tectonics, edited by J. Dewey and M. Coward, Geol. Soc. Spec. Publ., 28,349-408, 1987.

Holm, D. K., Relation of deformation and multiple intrusion in the Death Valley extended region, California, with implications for magma entrapment mechanism, J. Geophys. Res., 100, 10,495-10,505, 1995.

Holm, D. K., and R. K. Dokka, Major late Miocene cooling of the middle crust associated with extensional orogenesis in the Funeral Mountains, California, Geophys. Res. Lett., 18, 1775-1778, 1991.

Holm, D. K., and R. K. Dokka, Interpretation and tectonic implications of cooling histories: An example from the Black Mountains, Death Valley extended terrane, California, Earth Planet. Sci. Lett., 116, 63-80, 1993.

Holm, D. K., and B. P. Wernicke, Black Mountains crustal section, Death Valley extended terrain, California, Geology, 18, 520-523, 1990.

Holm, D. K., J. K. Snow, and D. R. Lux, Thermal and barometric constraints on the intrusive and unroofing history of the Black Mountains: Implications for timing, initial dip, and kinematics of detachment faulting in the Death Valley region, California, Tectonics, 11, 507-522, 1992.

Holm, D. K., J. W. Geissman, and B. P. Wernicke, Tilt and rotation of the footwall of a major normal fault system: Paleomagnetism of the Black Mountains, Death Valley extended terrane, California, Geol. Soc. Am. Bull., 105, 1373-1387, 1993.

Holm, D. K., T. L. Pavlish, and D. J. Topping, Black Mountains crustal section, Death Valley region, California, in Geologic Investigation of an Active Margin, Field Trip Guideb., pp. 31-54, Cordilleran Sect., Geol. Soc. of Am., Boulder, Colo., 1994a.

Holm, D. K., R. J. Fleck, and D. R. Lux, The Death Valley Turtlebacks reinterpreted as Miocene-Pliocene folds of a major detachment surface, J. Geol., 102, 718-727, 1994b.

Hornafius, J. S., B. P. Luyendyk, and R. R. Terres, Timing and extent of Neogene tectonic rotation in the western transverse ranges, California, Geol. Soc. Am. Bull., 97, 1476-1487, 1986.

Hudson, M. R., and J. W. Geissman, Paleomagnetic and structural evidence for middle Tertiary counterclockwise rotation in the Dixie Valley region, west-central Nevada, Geology, 15, 638-642, 1987.

Hudson, M. R., J. G. Rosenbaum, C. S. Gromme, R. B. Scott, and P. D. Rowley, Paleomagnetic evidence for counterclockwise rotation in a broad sinistral shear zone, Basin and Range province, southeastern Nevada and southwestern Utah, in Accommodation Zones and Transfer Zones: The Regional Segmentation of the Basin and Range, edited by J. E. Faulds and J. H. Stewart, Spec. Pap. Geol. Soc. Am., 323, 149-180, 1998.

Hunt, C. B., and D. R. Mabey, Stratigraphy and structure, Death Valley, California, U.S. Geol. Surv. Prof. Pap., 494-A, 162 pp., 1966.

Janecke, S. U., J. W. Geissman, and R. L. Bruhn, Localized rotation during Paleogene extension in east central Idaho: Paleomagnetic and geologic evidence, Tectonics, 10, 403-432, 1991.

Kirschvink, J. L., The least squares line and plane analysis of paleomagnetic data, Geophys. J. R. Astron. Soc., 62, 699-718, 1980.

Knott, J. R., A. M. Sarna-Wojcicki, C. E. Meyer, J. C. Tinsley, III, S. G. Wells, and E. Wan, Late Cenozoic stratigraphy and tephrochronology of the western Black Mountains piedmont, Death Valley, California: Implications for the tectonic development of Death Valley, in Cenozoic Basins of the Death Valley Region, edited by L. A. Wright and B. W. Troxel, Spec. Pap. Geol. Soc. Am., 333, 345366, 1999.

Labotka, T. C., and A. L. Albee, Late Precambrian depositional environment of the Pahrump Group, Panamint Mountains, Spec. Pap. 129, pp. 93-100, Calif. Div. of Mines and Geol., Sacramento, 1988.

Mancktelow, N. S., and T. L. Pavlis, Fold-fault relationships in low-angle detachment systems, Tectonics, 13, 668-685, 1994.

Mankinen, E. A., E. Larson, C. S. Gromme, M. Preveot, and R. S. Coe, The Steen Mountain (Oregon) geomagnetic polarity transition, 3, Its regional significance, J. Geophys. Res., 92, 8057-8076, 1987.

McFadden, P. L., and F. J. Lowes, The discrimination of mean directions drawn from Fisher distributions, Geophys. J. R. Astron. Soc., 67, 19-33, 1981 .

McFadden, P. L., and M. W. McElhinny, Classification of the reversal test in paleomagnetism, Geophys. J. Int., 103, 725-729, 1990.

McFadden, P. L., R. T. Merrill, M. W. McElhinny, and S. Lee, Reversals of the Earth's magnetic field and temporal variations of the dynamo families, J. Geophys. Res., 96, 3923-3933, 1991.

McKenna, L. W. III, New Rb-Sr constraints on the age of detachment faulting in the Panamint Range, Death Valley California, Geol. Soc. Am. Abstr. Programs, 18, 156, 1986.

McKenna, L. W. III, and K. V. Hodges, Constraints on middle MioceneRecent extension, Death Valley region, Basin and Range Province, Geol. Soc. Am. Abstr. Programs, 21, A353, 1989. 
McKenna, L. W. III, and K. V. Hodges, Constraints on the kinematics and timing of late Miocene-Recent extension between the Panamint and Black Mountains, southeastern California, in Basin and Range Extensional Tectonics at the Latitude of Las Vegas, Nevada, edited by B. P. Wernicke, Mem. Geol. Soc. Am., 176, 363-376, 1990.

McKenna, L. W. III, and L. W. Snee, Timing of movement and strain rate of Cenozoic extensional faulting in the Death Valley area, California, Geol. Soc. Am. Abstr. Programs, 22, A227, 1990.

Miller, M. G., Implications of ductile strain on the Badwater turtleback for pre-14-Ma extension in the Death Valley region, California, in Cenozoic Basins of the Death Valley Region, edited by L. A Wright and B. W. Troxel, Spec. Pap. Geol. Soc. Am., 333, 115126, 1999

Miller, M. G., and R. M. Friedman, Early Tertiary magmatism and probable Mesozoic fabrics in the Black Mountains, Death Valley, California, Geology, 27, 19-22, 1999.

Nelson, M. R., and C. H. Jones, Paleomagnetism and crustal rotation along a shear zone, Las Vegas Range, southern Nevada, Tectonics, 6, 13-34, 1987

Niemi, N. A., B. P. Wernicke, R. A. Brady, J. B. Saleeby, and G. C. Dunne, Distribution and provenance of the middle Miocene Eagle Mountain Formation, and implications for regional kinematic analysis of the Basin and Range province, Geol. Soc. Am. Bull., 113, 419-422, 2001.

Petronis, M. S., A reconnaissance paleomagnetic investigation bearing on vertical-axis rotation associated with a simple-shear transfer system in the Silver Peak Range, west-central Nevada, M.S. thesis, 273 pp., Univ. of N. M., Albuquerque, 1998.

Petronis, M. S., J. W. Geissman, D. K. Holm, B. P. Wernicke, and E. Schauble, Paleomagnetic data bearing on vertical-axis rotation within the central Death Valley Extended Terrane, Eos Trans. $A G U, 78(46)$, Fall Meet. Suppl., F189, 1997.

Rosenbaum, J. G., M. R. Hudson, and R. B. Scott, Paleomagnetic constraints on the geometry and timing of deformation at Yucca Mountain, Nevada, J. Geophys. Res., 96, 1963-1980, 1991.

Roy, J. L., and J. K. Park, The magnetization process of certain red beds: Vector analysis of chemical and thermal results, Can. J. Earth Sci., 11, 437-471, 1974.

Serpa, L., and T. L. Pavlis, Three-dimensional model of the late Cenozoic history of the Death Valley region, southeastern California, Tectonics, 15, 1113-1128, 1996.

Snow, J. K., and D. R. Lux, Tectono-sequence stratigraphy of Tertiary rocks in the Cottonwood Mountains and northern Death Valley area, California and Nevada, in Cenozoic Basins of the Death Valley Region, edited by L. A. Wright and B. W. Troxel, Spec. Pap. Geol. Soc. Am., 333, 17-64, 1999.

Snow, J. K., and A. R. Prave, Covariance of structural and stratigraphic trends: Evidence for anticlockwise rotation within the Walker Lane Belt Death Valley region, California and Nevada, Tectonics, 13, 712-724, 1994.

Snow, J. K., and B. P. Wernicke, Uniqueness of geological correlations: An example from the Death Valley extended terrane, Geol. Soc. Am. Bull., 101, 1351-1362, 1989 .

Snow, J. K., and B. P. Wernicke, Cenozoic tectonism in the central Basin and Range: Magnitude, rate, and distribution of upper crustal strain, $A m$. J. Sci., 300, 659-719, 2000.

Snow, J. K., J. W. Geissman, and B. P. Wernicke, Paleomagnetic data from Paleozoic sedimentary rocks and Permo-Triassic intrusions, Death Valley area, southeast California: Implications for Mesozoic and Cenozoic Deformation, Eos Trans. AGU, 74(43), Fall Meet. Suppl., 206, 1993.

Sonder, L. J., C. H. Jones, S. L. Salyards, and K. M. Murphy, Vertical axis rotations in the Las Vegas shear zone, southern Nevada: Paleomagnetic constraints on kinematics and dynamics of block rotations, Tectonics, 13, 769-788, 1994.

Stamatakos, J. A., D. A. Ferrill, and K. H. Spivey, Paleomagnetic constraints on the tectonic evolution of Bare Mountain, Nevada, Geol. Soc. Am. Bull., 110, 1530-1546, 1998 .

Stewart, J. H., Extensional tectonics in the Death Valley area, California: Transport of the Panamint Range structural block $80 \mathrm{~km}$ northwestward, Geology, 11, 153-157, 1983.
Stewart, J. H., J. P. Albers, and F. G. Poole, Summary of regional evidence for right-lateral displacement in the western Great Basin, Geol. Soc. Am. Bull., 79, 1407-1413, 1968.

Thompson, R. A., M. E. Milling, R. J. Fleck, L. A. Wright, and N. W. Rogers, Temporal, spatial, and compositional constraints on volcanism associated with large-scale crustal extension in Central Death Valley, California, Geol. Soc. Am. Abstr. Programs, 74, 624, 1993.

Topping, D. J., Paleogeographic reconstruction of the Death Valley extended region: Evidence from Miocene large rock-avalanche deposits in the Amargosa Chaos basin, California, Geol. Soc. Am. Bull., 105, 1190-1213, 1993.

Van der Voo, R., Paleomagnetism of the Atlantic, Tethys, and Iapetus Oceans, Cambridge Univ. Press, New York, 1993.

Wawryzniec, T. F., J. W. Geissman, and R. E. Anderson, Paleomagnetic data bearing on style of Miocene deformation in the Lake Mead area, southern Nevada, J. Struct. Geol., 23, 1255-1279, 2001.

Wernicke, B. P., Cenozoic extensional tectonics of the U.S. Cordillera, in The Geology of North America, vol. G-3, The Cordilleran Orogen: Conterminous U.S., edited by B. C. Burchfiel, P. W. Lipman, and M. L. Zoback, pp. 553-583, Geol. Soc. of Am., Boulder, Colo., 1992.

Wernicke, B. P., A new measurement of Cenozoic crustal extension across the Death Valley region, Basin and Range Province, Geol. Soc. Am. Abstr. Programs, 25, A352, 1993.

Wernicke, B. P., J. D. Walker, and K. P. Hodges, Field guide to the northern part of the Tucki Mountain fault system, Death Valley region, California, in This Extended Land: Geology Journeys in the Southern Basin and Range, edited by D. L. Weide, Spec. Publ. 2, pp. 255-267, Geosci. Dep., Univ. of Las Vegas, Las Vegas, Nev., 1988a.

Wernicke, B. P., G. J. Axen, and J. K. Snow, Basin and Range extensional tectonics at the latitude of Las Vegas, Nevada, Geol. Soc. Am. Bull., 100, $1738-1757,1988 \mathrm{~b}$

Wright, L. A., Late Cenozoic fault patterns and stress fields in the Great Basin and westward displacement of the Sierra Nevada block, Geology, 4, 489-494, 1976.

Wright, L. A., Geology of the northern half of the Confidence Hills 15minute Quadrangle, Death Valley region, eastern California: The area of the Amargosa chaos, Map Sheet 34, scale 1:24,000, p. 31, Calif. Div. of Mines and Geol., Sacramento, 1981.

Wright, L. A., and B. W. Troxel, Shallow-fault interpretation of the Basin and Range structures, southwestern Great Basin, in Gravity and Tectonics, edited by K. A. De Jong and R. Scholten, pp. 397-407, John Wiley, New York, 1973.

Wright, L. A., B. W. Troxel, and R. E. Drake, Contrasting space-time patterns of extension-related, Late Cenozoic faulting, southwestern Great Basin, Geol. Soc. Am. Abst. Programs, 15, 287, 1983.

Wright, L. A., R. E. Drake, and B. W. Troxel, Evidence for the westward migration of severe Cenozoic extension, southeastern Great Basin, California, Geol. Soc. Am. Abstr. Programs, 16, 701, 1984.

Wright, L. A., R. A. Thompson, B. W. Troxel, T. L. Pavlis, E. H. DeWitt, J. K. Otton, M. A. Ellis, M. G. Miller, and L. F. Serpa, Cenozoic magmatic and tectonic evolution of the east-central Death Valley region, California, in Geological Excursions in Southern California and Mexico, Field Trip Guideb., edited by M. J. Walawender and B. B. Hanan, pp. 93-127, Geol. Soc. of Am., Boulder, Colo., 1991.

Wright, L. A., R. C. Greene, I. Cemen, F. C. Johnson, A. R. Prave, Tectonostratigraphic development of the Miocene-Pliocene Furnace Creek Basin and related features, Death Valley region, California, in Cenozoic Basins of the Death Valley Region, edited by L. A. Wright and B. W. Troxel, Spec. Pap. Geol. Soc. Am., 333, 87-114, 1999.

J. W. Geissman and M. S. Petronis, Department of Earth and Planetary Sciences, University of New Mexico, 203 Northrop Hall, Albuquerque, NM 87131-1116, USA. (jgeiss@unm.edu; mspetro@unm.edu)

D. K. Holm, Department of Geology, Kent State University, Kent, OH 44242, USA. (dholm@kent.edu)

E. Schauble and B. Wernicke, Division of Geological and Planetary Sciences, California Institute of Technology, MC 100-23, Pasadena, CA 91125, USA. (edwin@gps.caltech.edu; brian@gps.caltech.edu) 